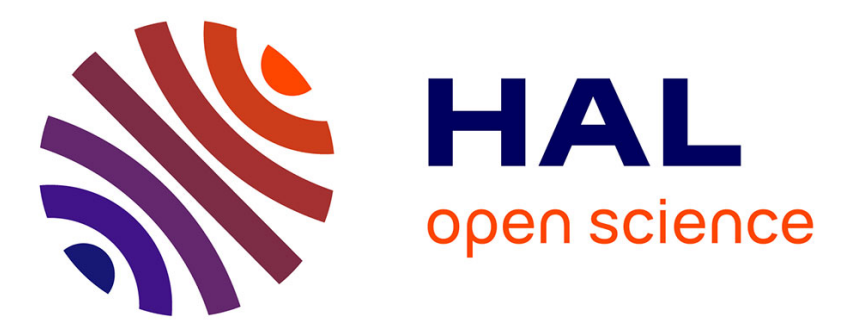

\title{
Passive Chaos Bandwidth Enhancement Under Dual-Optical Feedback with Hybrid III-V/Si DFB Laser
}

Kevin Schires, Sandra Gomez, Antonin E Gallet, Guang-Hua Duan, Frederic Grillot

\section{- To cite this version:}

Kevin Schires, Sandra Gomez, Antonin E Gallet, Guang-Hua Duan, Frederic Grillot. Passive Chaos Bandwidth Enhancement Under Dual-Optical Feedback with Hybrid III-V/Si DFB Laser. IEEE Journal of Selected Topics in Quantum Electronics, 2017, 23 (6), pp.1801309. 10.1109/JSTQE.2017.2732830 . hal-02101630

\section{HAL Id: hal-02101630 \\ https://hal.science/hal-02101630}

Submitted on 17 Apr 2019

HAL is a multi-disciplinary open access archive for the deposit and dissemination of scientific research documents, whether they are published or not. The documents may come from teaching and research institutions in France or abroad, or from public or private research centers.
L'archive ouverte pluridisciplinaire HAL, est destinée au dépôt et à la diffusion de documents scientifiques de niveau recherche, publiés ou non, émanant des établissements d'enseignement et de recherche français ou étrangers, des laboratoires publics ou privés. 


\title{
Passive Chaos Bandwidth Enhancement Under Dual-Optical Feedback with Hybrid III-V/Si DFB Laser
}

\author{
Kevin Schires, Sandra Gomez, Antonin Gallet, Guang-Hua Duan, Senior Member, IEEE, and Frédéric Grillot
}

\begin{abstract}
The chaotic dynamics of a DFB laser are studied experimentally under a combination of short and long feedbacks. Chaos bandwidth enhancement is demonstrated using a hybrid III-V/Si DFB laser with a large relaxation oscillation frequency (ROF) of $14 \mathrm{GHz}$. The impact of short feedback on the ROF is studied and an increase of $2 \mathbf{G H z}$ is observed. Under long feedback, the route to chaos of the device and its dependence on the short feedback dynamics are studied. The short feedback allows tuning the chaotic dynamics obtained under long feedback, and the increase of the ROF translates into an enhancement of the chaos bandwidth to above $16 \mathrm{GHz}$. This configuration can allow generation of wideband chaos using a single laser source in a photonic integrated circuit.
\end{abstract}

Index Terms-III-V materials, nonlinear dynamics, optical feedback, silicon photonics, secure communications.

\section{INTRODUCTION}

O ILICON photonics offer tight integration of a variety of active and passive optical and electrical components, and gained so much interest in the last decade that it is now considered one of the most promising technology for optical applications [1], [2]. Building on the mature fabrication techniques first developed for microelectronics allows creating photonic integrated circuits (PICs) with a high density of optical components, in high volumes and at low costs. Academic and industrial efforts led to the development of novel technical solutions for a variety of domains including sensing, measurement instrumentation, optical signal processing and telecommunications

Manuscript received February 7, 2017; revised March 30, 2017 and June 29, 2017; accepted June 30, 2017. This work was supported by the European Union Horizon 2020 Programme under the PICs4All Project (http://www.pics4all.jeppix.eu). (Corresponding author: Kevin Schires.)

K. Schires and S. Gomez are with Télécom ParisTech, Université Paris-Saclay, Paris 75634, France (e-mail: schires@enst.fr; sandra.gomez@ telecom-paristech.fr).

A. Gallet is with Télécom ParisTech, Université Paris-Saclay, Paris 75634, France, and also with the III-V Lab, Joint Laboratory of Nokia Bell Labs, Thales Research and Technology, and CEA-Leti, Fresnel 91767, France (e-mail: antonin.gallet@3-5lab.fr).

G.-H. Duan are with the III-V Lab, Joint Laboratory of Nokia Bell Labs, Thales Research and Technology, and CEA-Leti, Fresnel 91767, France (e-mail: guanghua.duan@3-5lab.fr).

F. Grillot is with Télécom ParisTech, Université Paris-Saclay, Paris 75634, France, and also with the Center for High Technology Materials, University of New Mexico, Albuquerque, NM 87106-4343 USA (e-mail: grillot@ telecom-paristech.fr).

Color versions of one or more of the figures in this paper are available online at http://ieeexplore.ieee.org.

Digital Object Identifier 10.1109/JSTQE.2017.2732830
[3], [4]. Recent advances in data centers and informatics [5] reveal how photonic integration will become increasingly used for data transmission, either inside a chip or for short-access and long-haul telecommunications networks [6], [7].

Each discrete component used in electro-optical devices had to be redesigned for integration on $\mathrm{Si}$, and a number of research works allowed developing novel integrated modulators, photodetectors, isolators, polarization controllers, amplifiers, as well as novel laser sources [8]. As laser cavities had to be adapted for integration into PICs, novel fabrication techniques were derived for the growth of the active material, and novel designs of the resonant cavity were proposed [9]-[12]. The active III-V material is generally not grown directly on a Si substrate but rather wafer bonded onto a Si waveguide: lattice mismatches between III-V compounds and $\mathrm{Si}$ as well as different thermal expansion lead to dislocation in Quantum Well (QW) materials and to poor device reliability. Novel Quantum Dot (QD) laser sources grown directly on Si have recently been reported [13], [14]: unlike QW materials, the localization of carriers in QDs make these less sensitive to defects. Complex laser cavities for single and multimode laser sources have been proposed [15], but such designs were shown to suffer from stability issues due to internal feedback sources [16] and a simpler DFB structure is studied in this work.

Multisection lasers have been demonstrated on PICs, and devices with an integrated external feedback cavity for use as chaotic emitters are of interest for both academic and industrial research [17]-[19]. PICs creating an external cavity with a phase section are important for research purposes as they allow study of the behavior of lasers under optical feedback with short cavities of controllable length, thus giving new insights into laser dynamics [20], [21]. Under optical feedback of increasing strength, the laser's relaxation oscillations become excited and lead to chaotic oscillation of the laser output [22]. PICs containing lasers with and external optical feedback cavity are thus very important for practical applications, as they allow the use of chaotic emitters in communication networks without imposing volume constraints for integration into existing emitter and receiver modules.

Two major applications of chaotic emitters in communication networks are random number generation and most importantly secure communications [23]. Chaotic communications rely on hiding data within a broad chaotic spectrum at the emitter level, and the data is recovered in the receiver. The bandwidth of the 
amplified feedback should thus be maximized to increase transmission rates while keeping the data secure, and several schemes were proposed to obtain large chaos bandwidth, defined as the frequency under which $80 \%$ of the RF power is found [24]. Passive enhancement of the chaos bandwidth was proposed using phase-conjugate feedback and a high-reflectivity mirror [25]. Active solutions using optical injection from two laser sources in addition to optical feedback demonstrated chaos bandwidths as high as $32 \mathrm{GHz}$ [26].

Another way of generating broad chaos is to increase the relaxation oscillation frequency (ROF) of the laser source. Under optical feedback, the generated chaotic spectra scale with the relaxation frequency of the laser, as chaotic dynamics build up from enhanced relaxation oscillations. In addition, the ROF can be tuned using optical feedback, depending on the feedback phase and strength [22], [27], [28], and that dynamics can be generated by tailoring the feedback and controlling its phase [29]. It was demonstrated that PICs with an integrated amplified external cavity with a phase section allowed forcing self-pulsations in the laser at a frequency tuneable to above $40 \mathrm{GHz}$ [30], [31].

In this article, we study the enhancement of the bandwidth of chaotic dynamics using a combination of two passive cavities of different scales. Chaos bandwidth enhancement is demonstrated using a hybrid III-V/Si QW DFB laser in order to prove applications to PICs. As the optical cavities are created in a fibered setup and not within the chip, only low feedback strengths are achieved. This study shows for the first time the evolution of the dynamics of a hybrid III-V/Si DFB laser with the strength of a long feedback or the phase of a short feedback, and combinations of the two. In Section I, the device is presented and characterized, and the impact of internal reflections on laser operation is discussed. The free-running laser exhibits high relaxation oscillations of $14 \mathrm{GHz}$ which make it ideal for this study as large ROF simplifies the access to wide chaos bandwidths. The route to chaos of the hybrid laser under long feedback is studied in Section III, and compared with that of a commercial III-V DFB laser, showing similar behaviors between the two types of laser despite significant differences in their structures. In Section IV, the laser is subject to a short feedback cavity which allows increasing its ROF by $2 \mathrm{GHz}$. In Section V, the laser is subject to both feedback cavities, and the chaos bandwidth is found to be enhanced in the same fashion as the ROF. The chaos bandwidth is increased by up to $13 \%$ and reaches a maximum of $16.4 \mathrm{GHz}$, showing that this combination of two feedbacks allows controlling the bandwidth and characteristics of the generated chaos despite the very low feedback strength allowed by the setup. Such passive chaos bandwidth enhancement can be applied to standard III-V DFB lasers, and the setup is transposable to a PIC constituted of the laser and two cavities with optional phase sections, allowing wide chaos generation with low power consumption.

\section{EXPERIMENTAL APPARATUS}

\section{A. Device Studied}

Fig. 1 presents a schema of the DFB structure studied. The $1 \mathrm{~mm}$-long hybrid III-V Silicon-on-Insulator (SOI) device is

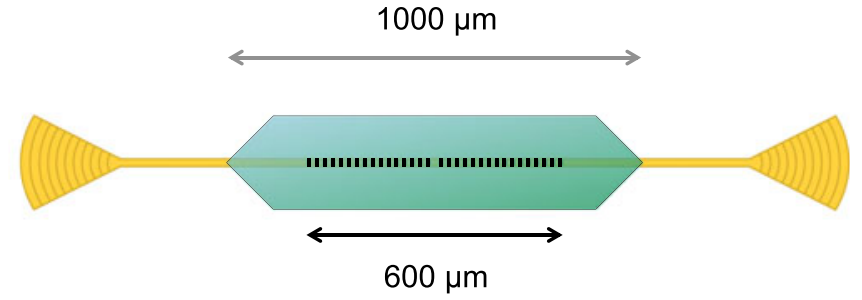

(a)

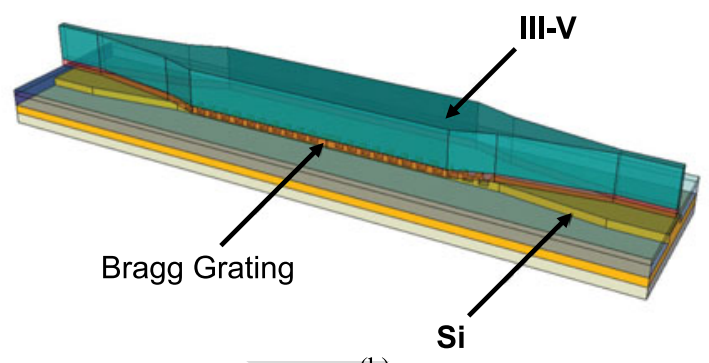

(b)

Fig. 1. (a) Schematic and (b) structure of the device studied, showing the $\mathrm{Si}$ waveguide (yellow) and the III-V material (green). The vertical couplers are represented in (a) at both extremities of the Si waveguide.

fabricated using a III-V QW active medium bonded on top of 132 the processed silicon waveguide, and constituted of a DFB laser 133 with tapers on each side [15]. To ensure single-mode operation, 134 a $50 \mathrm{~nm}$-deep and $600 \mu \mathrm{m}$-long Bragg grating with a quarter- 135 wavelength phase shift in the center is etched on the silicon 136 waveguide. The strength of the grating is chosen such that the 137 product $\kappa L_{\text {bragg }}$ is of a few units, and the period of the grating 138 is of $240 \mathrm{~nm}$. The light is coupled from the Si waveguide to the 139 III-V material with adiabatic tapers, and outcoupled using Verti- 140 cal Bragg Gratings (VBG) on both side of the device. The VBG 141 couples the light out of the laser with an angle of $80^{\circ}$ from the 142 waveguide, and light was thus coupled vertically using a fiber 143 positioned above the laser, with a $10^{\circ}$ angle from the normal 144 to the lasers surface. The VBG were only necessary for testing 145 purposes, in order to characterize several unprocessed devices 146 on the same bar, and in a PIC they would not be positioned after 147 the laser. They however affected our study in two ways: first via 148 their transmission losses of approximately $7 \mathrm{~dB}$, which will be 149 discussed later, and secondly by their parasitic reflectivity (be- 150 low $-23 \mathrm{~dB}$ ) which affected laser operation when biased high 151 above threshold.

\section{B. Characterisation}

Fig. 2(a) presents the evolution of the power coupled into an anti-reflection (AR)-coated lens-ended fiber with the bias current. All measurements presented in this paper were performed at $20{ }^{\circ} \mathrm{C}$. Around the threshold of about $45 \mathrm{~mA}$, the laser exhibits slight competition between two modes which translates into a kink in the curve. Between 50 and $150 \mathrm{~mA}$, very stable single-mode operation is observed with a side-mode suppression ratio above $50 \mathrm{~dB}$. Above 4 times threshold, the laser exhibited power drops which were also observed on the other lasers of the same bar, but differed from device to device. Such behavior hints that parasitic reflections are present within the devices: the vertical couplers and tapers create reflections which only 


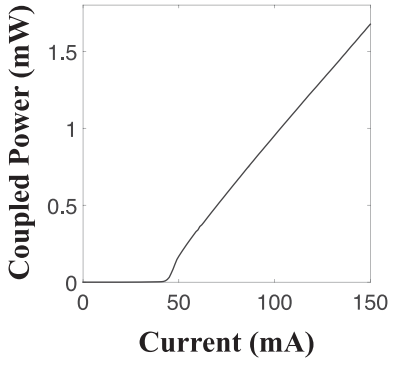

(a)

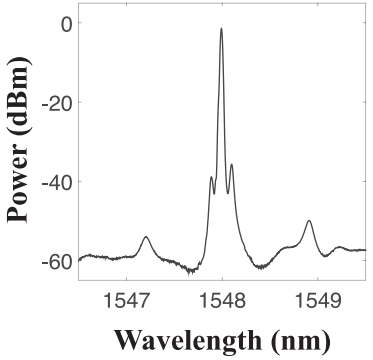

(b)

Fig. 2. (a) Evolution of the power coupled using an AR-coated lensed fiber with the pump current. (b) Optical spectrum at a bias current of $140 \mathrm{~mA}$.

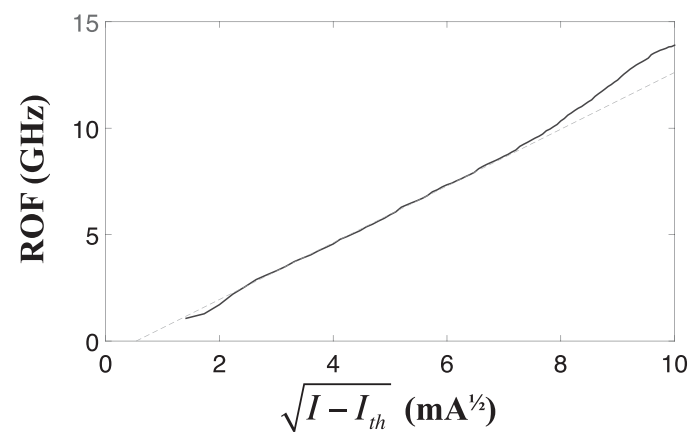

Fig. 3. Evolution of the ROF with the square root of the current overdrive above threshold.

seem to affect the laser far above threshold through variations of the optical power or changes in the ROF, as will be discussed next. As the amount of parasitic reflections varies from device to device, different sorts of power variation would indeed be expected between the different lasers. Fig. 2(b) presents the spectrum of the laser at $140 \mathrm{~mA}$, showing the well-suppressed side-modes as well as sidebands characteristic of relaxation oscillations. These spectra were measured using a Yenista OSA20 optical spectrum analyzer with a $20 \mathrm{pm}$ resolution, and these sidebands could be measured thanks to the rather high value of the ROF.

Fig. 3 presents the evolution of the ROF as a function of the square root of the current overdrive above threshold. It can be noted that very high frequencies are observed as the ROF reaches $14 \mathrm{GHz}$ at $150 \mathrm{~mA}$. Usually, due to gain compression, the power evolves in a sublinear fashion with increasing bias current, and the squared ROF follows the same trend. Here, it can be seen that the ROF rather evolves in the opposite way as it gradually increases above the linear fitting shown as a dashed line.

We believe that these high values of ROF stem from the use of Aluminium in the III-V compound forming the QW [32], but also from the aforementioned internal feedback. Under optical feedback, the ROF can oscillate around its free-running value depending on the feedback strength and delay [22]. Here, the internal feedback conditions thus allow a small enhancement of the ROF for some bias currents, showing an effect of parasitic feedback very different from the detrimental impact it can have further above threshold.

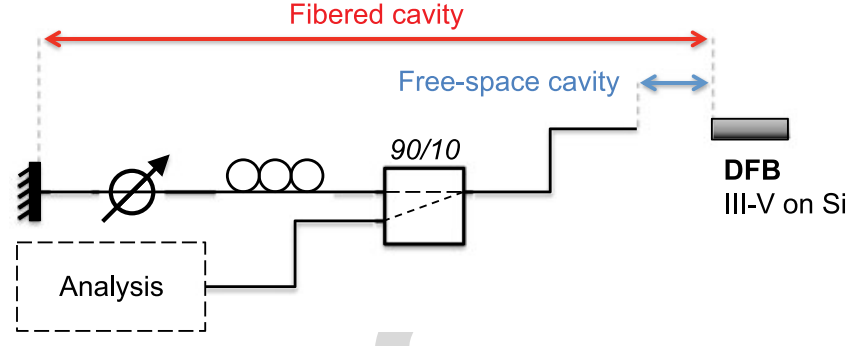

Fig. 4. Experimental setup allowing combination of short and long feedback.

\section{Experimental Setup}

Fig. 4 presents the experimental setup used for the following measurements [16]. The DFB is kept at a bias current of $146 \mathrm{~mA}$ and at $20^{\circ} \mathrm{C}$. The light of the DFB laser is coupled using either an AR-coated lensed fiber or a cleaved uncoated one. The cleaved fiber allows creating a short free-space feedback cavity [33] of the order of $100 \mu \mathrm{m}$, re-injecting about $3 \%$ of the light back into the device with a time delay below 1 ps. On the other hand, the lens-ended fiber minimizes such reflections and only allows light from the setup to be re-injected into the device. In the fiberized setup, a 90/10 splitter is used to create a feedback path consisting of a polarization controller and a Yenista BackReflector (BKR) module, equivalent to a mirror with variable losses. This long fibered cavity measures approximately $7 \mathrm{~m}$ and allows re-injecting at most $8 \%$ of the light into the device. Note that due to the transmission losses of the vertical couplers, a difference must be made between the light re-injected into the component and the light that reaches the laser cavity. The maximum feedback strengths considering the light that reaches the laser cavity are thus of $0.1 \%$ and $0.3 \%$ for the short and long cavities, respectively.

Using either fiber and controlling the attenuation of the long feedback path allows studying the laser into the following four situations: free-running with only parasitic reflections from the setup, under short (free-space) feedback only, under long (fibered) feedback only, and under a combination of both feedbacks. For the short feedback, while the feedback strength is fixed by the refractive index of the fiber, the phase can be tuned using a piezoelectric actuator allowing gradually moving the fiber towards or away from the device. Concerning the long feedback, while the feedback phase can be changed the same way it does not impact laser behavior given the large external cavity length. Feedback strength can however be tuned by changing the attenuation of the BKR.

\section{LONG FEEDBACK}

To assess the potential of such hybrid lasers as chaotic emitters, we first study the route to chaos that the devices follow under long feedback. The cavity used here is too long to represent the external cavity that would be integrated in a PIC, but it allows pushing the laser into chaotic operation where the phase of the long feedback has little impact on the dynamics. This will later allow us to dissociate the effect of the short and long feedbacks when combined together, as we can consider that the feedback phase will only affect the dynamics induced by the 


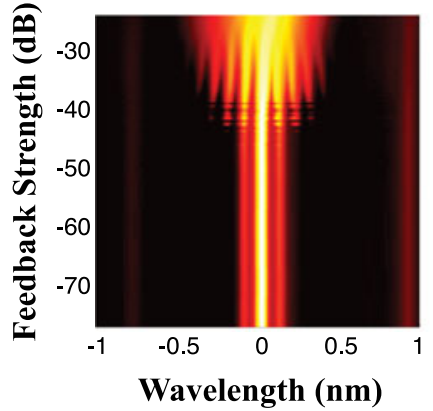

(a)

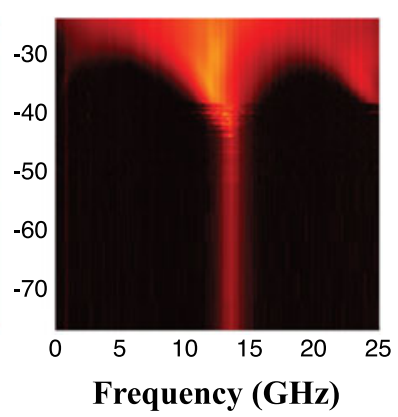

(b)
Fig. 5. Evolution of the (a) optical and (b) RF spectra with long feedback strength, using a lens-ended fiber.

short feedback. Fig. 5 presents the evolution of the optical and RF spectra with the long feedback strength, using the lensed fiber. The measurements performed with the lensed fiber have very little dependence on the position of the fiber and thus the feedback phase, as it will be shown later in the case of the freerunning laser. Routes to chaos under long feedback were thus found to be identical for different positions of the fiber.

The route observed is typical of a DFB laser under long optical feedback despite major differences in the device structures: first of all, the hybrid DFB is constituted of two evanescently coupled waveguides, one passive and one active, unlike the standard III-V DFB. In addition, in the hybrid device the tapered regions on each side of the DFB structure may slightly amplify the light fed back into the laser, or even act as source of internal reflections [16]. The proposed chaos bandwidth enhancement method could also be applied to a standard III-V DFB, for which higher feedback strengths could be achieved. Demonstrating the changes in ROF and bandwidth in the case of hybrid lasers is however interesting, as it shows that the complexity of the lasers used in PICs does not necessarily affect their behavior under optical feedback.

For very low feedback strengths, the ROF can be seen at $14 \mathrm{GHz}$ in the RF spectrum. Around $-43 \mathrm{~dB}$ of feedback strength, temporally unstable periodic oscillations start to appear, and stabilize above $-40 \mathrm{~dB}$. These correspond to an excitation of relaxation oscillations that turn into chaotic dynamics above $-30 \mathrm{~dB}$ of feedback strength. Under maximum feedback strength, the bandwidth of the chaotic spectra is of $14.5 \mathrm{GHz}$.

Fig. 6 allows comparing the route to chaos of this hybrid DFB laser with that of a commercial Nokia III-V DFB laser. The laser is operated a three times its threshold where it has a ROF of $8 \mathrm{GHz}$. The routes appear to be very similar. Along the feedback strength axis, the routes appear to be shifted by $6 \mathrm{~dB}$ as the III-V DFB reaches chaotic operation for only $-36 \mathrm{~dB}$ of feedback strength. Note that in the case of the III-V laser, higher feedback strengths were achievable with the same setup as there were no extra losses between the fiber and the laser cavity. It can thus be seen that with the hybrid DFB we are only able to merely enter the chaotic regime, and that much broader spectra could be observed for stronger feedback. Along the wavelength axis, broader spectra are observed for the hybrid DFB in the region of periodic oscillations, as the base frequency of these oscillations

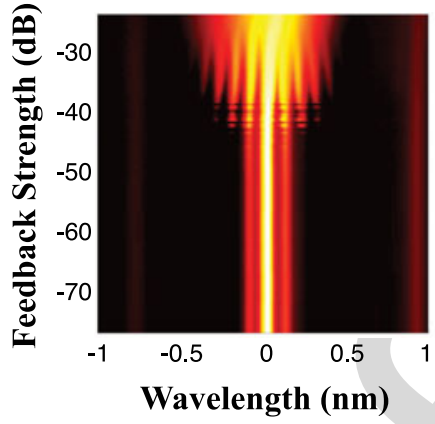

(a)

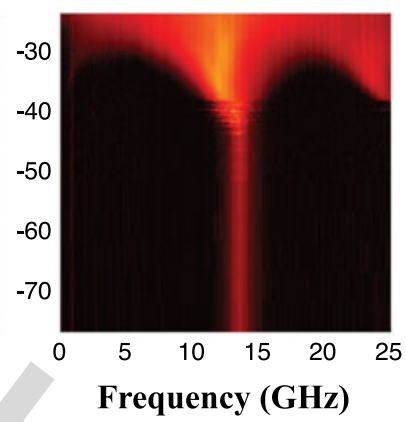

(b)
Fig. 6. Evolution of the optical spectra of a (a) III-V and (b) III-V/Si DFB laser with long feedback strength, using a lens-ended fiber.

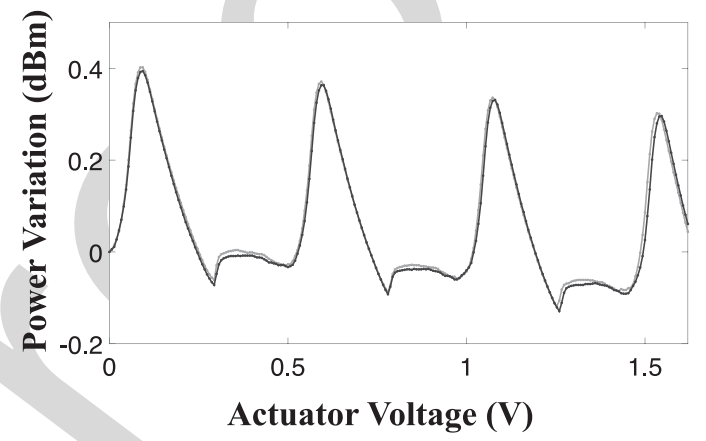

Fig. 7. Variation of the power coupled with the cleaved fiber as a function of the voltage applied to the piezoelectric actuator. Two measurements for different fiber positions are presented in black and grey.

is the ROF, and we expect that the spectra observed for the III-V DFB could be obtained for the hybrid one, broadened by a factor close to the ratio between the two ROFs.

\section{SHORT FEEDBACK}

Using the cleaved fiber and setting the long feedback strength 285 to its minimum of $-79 \mathrm{~dB}$ allows studying the DFB under short 286 feedback only. Fig. 7 presents the variation of the coupled power 287 as a function of the voltage applied to the piezoelectric actuator. 288 As the coupling is only optimized for a voltage of $0 \mathrm{~V}$, the power 289 fades as the fiber is moved away from the device and the figure 290 appears skewed. A clear periodicity can however be observed 291 in the evolution of the power, as we are varying the phase of 292 the short feedback. For convenience, we choose as beginning of 293 each period the main peaks and consider that between each peak, 294 the phase is varied from 0 to $2 \pi$. Note that this does not represent 295 the absolute phase of the feedback, but only a representation 296 of the phase shift within a period.

Such variation of the output power under optical feedback 298 corresponds to the effect of a medium feedback strength and 299 usually exhibits bistability when the mirror is moved one way 300 or the other [22]. Fig. 8 presents a comparison between the 301 evolution of the power and spectrum when the fiber is moved in 302 both directions, as well as reference measurements performed 303 with the lensed fiber. The reference measurements shows the 304 weak impact of all parasitic reflections from the setup, and shows 305 that the cavity created by the cleaved fiber is mainly responsible 306 


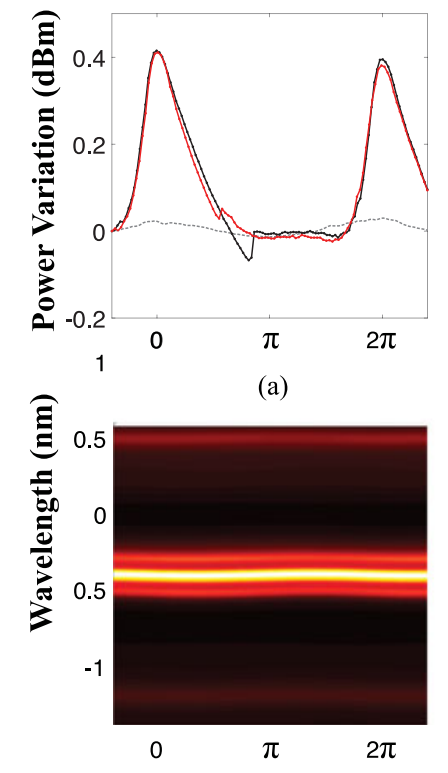

(b)

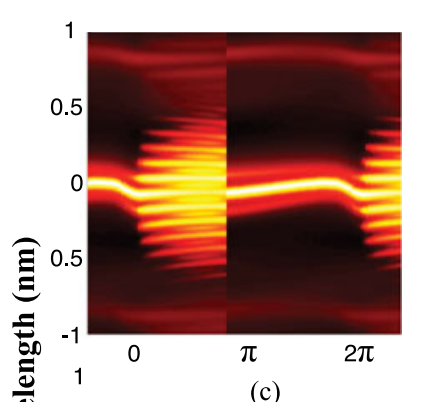

(c)

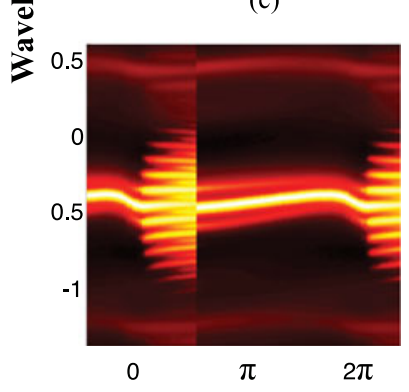

(d)

Fig. 8. (a) Variation of the power coupled with the cleaved fiber within one period when the fiber is moved away (black) or towards (red) the laser. The grey dashed line represents the power coupled when using the lensed fiber. (b) Evolution of the optical spectrum when using the lensed fiber. (c) and (d) Show the evolution of the optical spectrum when moving the fiber away or towards the laser, respectively.

for the power variation presented above. In Fig. 8(a) the gray dashed line shows that the power is oscillating sinusoidally with a low amplitude, which corresponds to the impact of weak feedback. Fig. 8(b) shows that the wavelength varies in a similar fashion. This confirms that any other variation of the power or wavelength is only due to the feedback created by the cleaved fiber. Fig. 8(a) also reveals a slight bistability in the evolution of the power, as the kinks observed after the peak occur for a phase shift of $0.3 \pi$ for one direction and $0.4 \pi$ for the other. While the bistability in the output power is negligible, it makes a significant difference in the optical spectrum. Note that all spectrum maps presented in this work are plotted in $\mathrm{dBm}$, with a logarithmic color scale.

Fig. 8(c) reveals that during each periods, the laser exhibits periodic dynamics between phases of 0 and $0.4 \pi$, with period doubling above $0.2 \pi$. When the kink occurs in the optical power, the laser suddenly stabilises, and it can be seen from the sidebands that the ROF changes with the phase. Fig. 8(d) shows that the same behaviour is observed when the fiber is moved in the opposite direction, thus leading to a wider region of stability. This wider region is of importance for this study as it appears that the largest ROFs are achieved within the bistablity window when the laser is stable, as this is where the mode's sidebands appear to be the farthest apart.

Fig. 9 shows the evolution of the optical and RF spectra of the DFB as the fiber is moved towards the laser, at a shorter distance. In order to clearly see the peak of the relaxation oscillations in the RF spectra, an EDFA set to a fixed output power of $10 \mathrm{dBm}$ was used to amplify the light before detection. A photodetector with a bandwidth of $30 \mathrm{GHz}$ and a Rohde \& Schwartz FSP

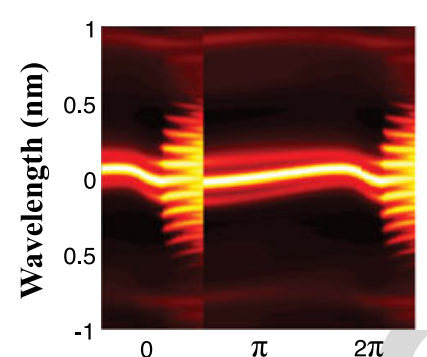

(a)

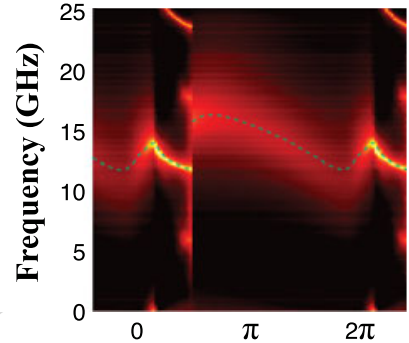

(b)
Feedback Phase (rad)

Fig. 9. Evolution of the (a) optical and (b) RF spectra within one period. The green dashed line shows the evolution of the ROF.

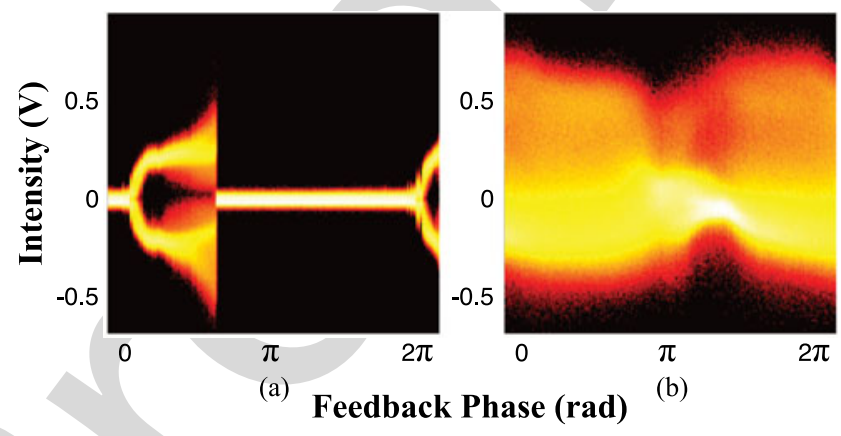

Fig. 10. Bifurcation diagrams as a function of the short feedback phase under (a) minimum and (b) maximum long feedback strength.

$40 \mathrm{GHz}$ electrical spectrum analyzer were used to perform the spectrum measurements. A slightly larger stability region is obtained, and the RF spectra show that within one period, the ROF varies between a minimum of $11 \mathrm{GHz}$ and a maximum of $16 \mathrm{GHz}$, thus allowing reaching frequencies $2 \mathrm{GHz}$ higher than the free-running ROF.

\section{COMBINATION OF FEEDBACKS}

Under long feedback only, the hybrid DFB exhibits a classic route to chaos where chaotic dynamics are obtained from the excitation of relaxation oscillations. Under short feedback only, it is possible to tune the value of the ROF while keeping the laser into stable operation. Combining both feedbacks will allow us to study whether the enhancement of the ROF under short feedback can be used to generate broader chaos by first tailoring the ROF and then exciting relaxation oscillations into chaotic ones. In this section, the laser is thus subjected to a combination of short and long feedbacks by using the cleaved fiber and varying the long feedback strength.

Fig. 10 presents the bifurcation diagrams of the dynamics as a function of the phase of the short feedback, under minimum and maximum long feedback strengths. Under minimum long feedback strength, the diagram clearly shows the apparition of the periodic oscillations and the sudden transition from oscillating to stable operation. Under maximum feedback, it can be seen that the chaotic signal changes significantly with the short feedback phase.

Fig. 11 presents the evolution of optical and RF spectra with the position of the cleaved fiber for four levels of long feedback 

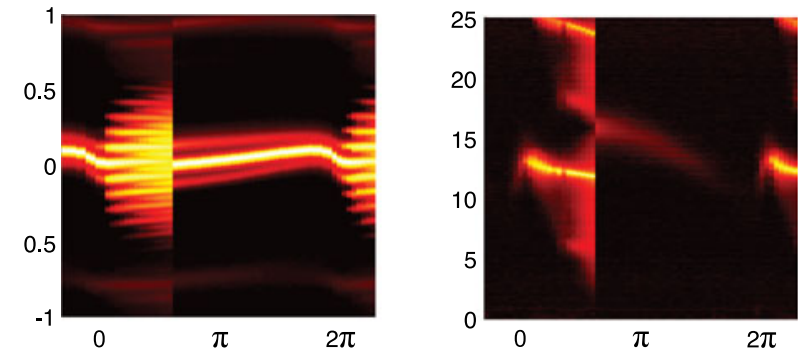

(a)

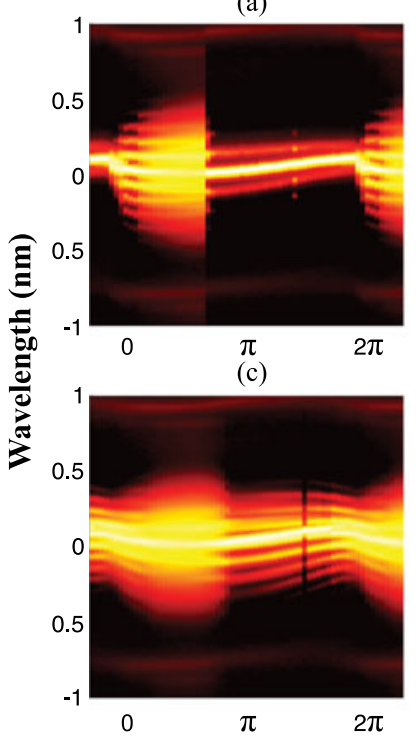

(e)

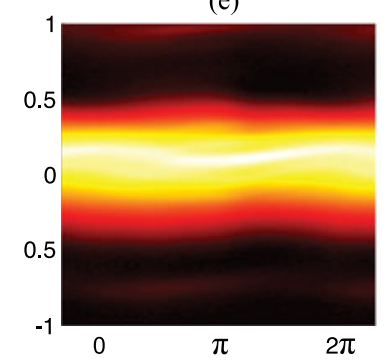

(g)

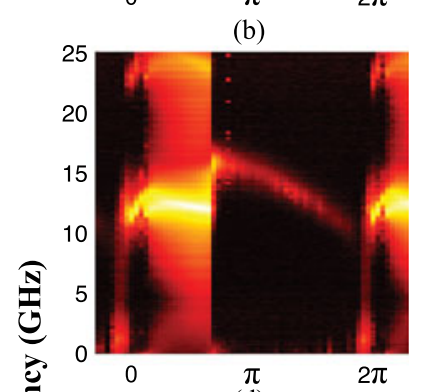

(d)

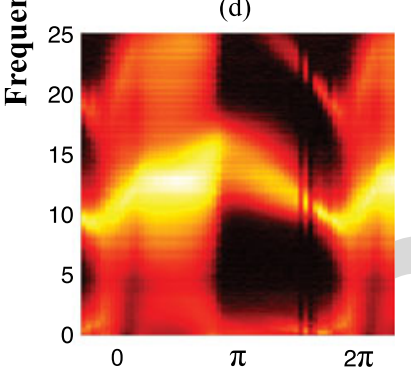

(f)

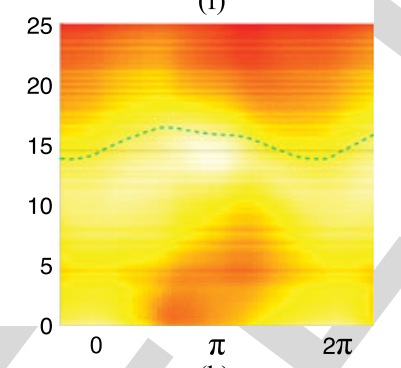

Fig. 11. Evolution of optical (left) and RF (right) spectra with the position of the cleaved fiber for long feedback strengths of (a), (b) $-79 \mathrm{~dB}$, (c), (d) $-42 \mathrm{~dB}$, (e), (f) $-36 \mathrm{~dB}$ and $(\mathrm{g}),(\mathrm{h})-26 \mathrm{~dB}$. In (h) the black line shows the chaos bandwidth under long feedback only, and the green dashed line shows the evolution of chaos bandwidth with the phase of the short feedback.

strength: the minimum of $-79 \mathrm{~dB},-42 \mathrm{~dB},-36 \mathrm{~dB}$ and the maximum of $-26 \mathrm{~dB}$. No optical amplification was used for the RF spectrum measurements. The route towards chaos under long feedback clearly changes with the short feedback phase, as periodic dynamics appear for different long feedback strengths at the different positions of the cleaved fiber. Regions where periodic oscillations occur under short feedback alone appear to enter chaotic operation first. The sharp transition between oscillating and stable operation gradually disappears as the long feedback strength is increased. In a similar way as in Figs. 5 and 6 , some points exhibit temporally unstable dynamics for feedback phases between $1.3 \pi$ and $1.5 \pi$ and feedback strengths of -42 and -36 , which can be seen as a disappearance of the

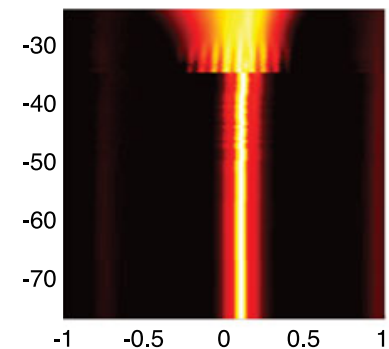

(a)

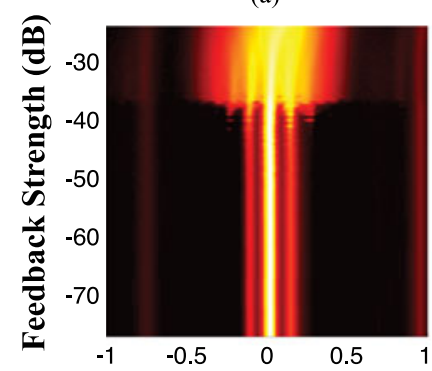

(c)

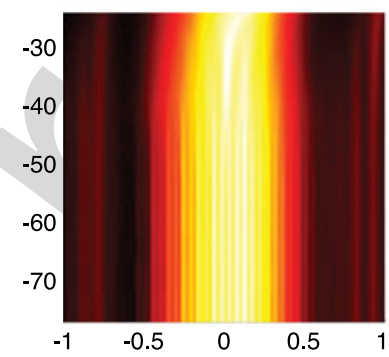

(e)

Wavelength (nm)

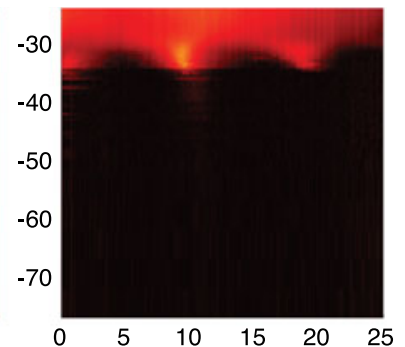

(b)

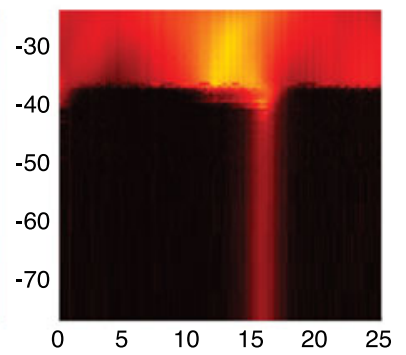

(d)

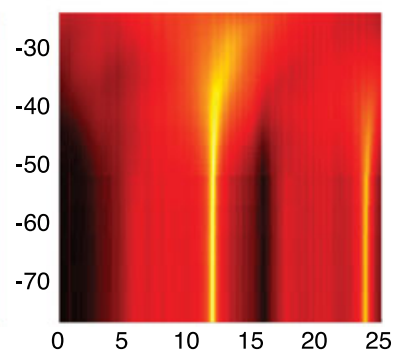

(f)

Frequency (GHz)
Fig. 12. Evolution of the optical (left) and RF (right) spectra with the long feedback strength for three positions of the cleaved fiber. (a) and (b) correspond to a feedback phase of $1.9 \pi$ (minimum ROF, stable). (c) and (d) correspond to a feedback phase of $0.3 \pi$ (maximum ROF, stable). (e) and (f) correspond to a feedback phase of $0.25 \pi$ (strongest oscillations).

dynamics for some isolated feedback phases. It is interesting 378 to observe that, as the long feedback strength is increased, the 379 wavelength of the spectrum peak oscillates in a more and more 380 sinusoidal fashion, such that no bistability was observed under 381 maximum long feedback strength.

The long feedback strength necessary to reach chaotic operation seems to depend on the short feedback phase, and it can be seen in Fig. 11(g) and (h) that the width of the chaotic spectrum varies with the feedback phase too. In Fig. 11(h), the superimposed green dashed line shows the evolution of the chaos bandwidth with the short feedback phase. The black dotted line shows as a reference the bandwidth of the chaos measured under maximum long feedback only, corresponding to the spectrum under maximum long feedback strength in Fig. 5(b). With the addition of short feedback, the chaos bandwidth oscillates between 13.7 and $16.4 \mathrm{GHz}$ by following very closely the evolution of the ROF in Fig. 9(b). Minimum and maximum chaos bandwidth are indeed respectively found close to the feedback phases where minimum and maximum ROF are observed in the absence of long feedback. 


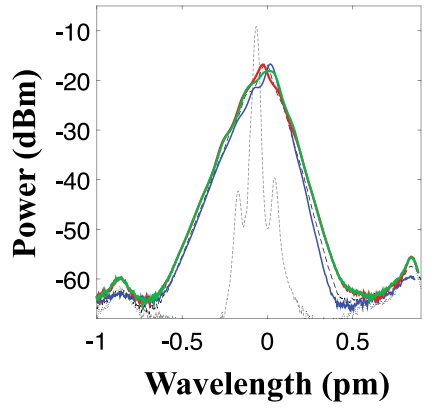

(a)

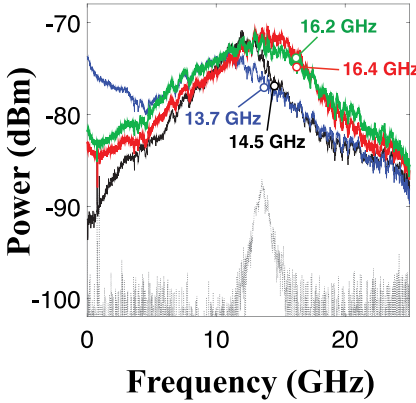

(b)

Fig. 13. Optical (left) and RF (right) spectra under maximum long feedback and their bandwidth, the dashed lines showing the free-running spectra as reference. The black curve corresponds to maximum long feedback alone. The blue (resp. red) curve corresponds to both feedbacks with minimum (maximum) ROF. The green curve corresponds to both feedback with strongest periodic oscillations.

Three specific routes to chaos are thus of interest: those where short feedback does not destabilize the laser but induces either a reduction of the $\mathrm{ROF}$ to its minimum value of $11 \mathrm{GHz}$ (phase of $1.9 \pi$ ) or an increase to its maximum value of $16 \mathrm{GHz}$ (phase of $0.3 \pi$ ), and one where short feedback induces the strongest periodic oscillations (phase of $0.25 \pi$ ). Fig. 12 presents these three routes. In the first two routes, the laser remains stable until slightly higher feedback strengths than compared to Fig. 5(a). The window where periodic oscillations are observed is however very narrow, as chaos appears around the same feedback strength of about $-30 \mathrm{~dB}$. In the last route, chaotic dynamics seem to appear for a lower long feedback strength of $-38 \mathrm{~dB}$, but under maximum long feedback the end of the route appears similar to that in Fig. 12(d). This is however not surprising given that the last two routes are measured for short feedback phases that are rather close, and that no discontinuity can be seen in Fig. 11(g) and $(h)$ between these phases.

The optical and RF spectra obtained under maximum long feedback for these three operating conditions are shown in Fig. 13, along with the free-running spectra and the chaotic ones obtained under maximum long feedback alone. Fig. 13(b) shows that the chaotic spectrum obtained for a feedback phase of $1.9 \pi$ leads to the minimum bandwidth of $13.7 \mathrm{GHz}$ observed in Fig. 11(h). Both the other routes lead to a bandwidth of 16.2 $\mathrm{GHz}$, despite slight differences in the chaotic spectra.

\section{CONCLUSION}

A combination of long and short feedback are used to respectively generate chaotic dynamics in a DFB laser and tune the chaos bandwidth. The dynamics of a hybrid III-V/Si DFB laser were studied under such combination of feedbacks to show the possibility to achieve passive chaos bandwidth enhancement in PICs. The routes of the dynamics are extensively characterized by varying the phase of the short feedback and strength of the long one. Owing to the large ROF of the free-running laser, chaotic dynamics with a bandwidth of $14.5 \mathrm{GHz}$ can be generated using a long external feedback cavity. The route to chaos appears to be very similar to that of a standard commercial III-V DFBs, but due to the transmission losses of the device's vertical couplers, maximum feedback strengths of only $0.3 \%$ were achievable. The impact of a short external cavity on the laser dynamics revealed a variation of the ROF of $5 \mathrm{GHz}$, with a maximum value of $16 \mathrm{GHz}$. When combining short and long feedbacks, this $2 \mathrm{GHz}$ increase of the ROF translates into a similar increase of the chaos bandwidth, which reaches $16.4 \mathrm{GHz}$.

The tuneability of the chaotic dynamics and of their bandwidth is of interest for applications requiring random number generation, or broad chaos generation. With feedback cavities integrated into a Si waveguide along with a phase section, higher feedback strengths would be achievable for both short and long feedbacks. Chaotic spectra much wider than those reported in this work could thus be obtained using a single component and passive optical cavities with optional phase sections. This would be of prime importance for the development of low-consumption integrated chaotic transmitters and receivers for secure communications.

As the wide bandwidth relies primarily on the large ROF of the laser, the design of the device is extremely important, and for these applications QW sources may be more appealing than QD sources as QW lasers generally exhibit much higher ROFs than QD ones. In the case of application in a Si PIC, the design of the $\mathrm{Si}$ waveguides would be very important too, as all the possible sources of reflections within the PIC will affect the dynamics of the laser. In this work, at least two feedback cavities allow pushing the ROF towards the values reported here: the cavity created by the cleaved fiber, but also the internal parasitic sources of feedback which seem to increase the ROF of the free-running laser already. If sources of feedback are inevitable in a PIC, they can thus be designed to potentially enhance the laser's operation instead of hindering it.

Future work will focus on the simulation of single mode III-V and III-V/Si lasers subject to two external cavities in order to attempt to replicate these experimental results. Conditions to maximize the increase of the ROF under short feedback will thus be studied in the presence of several short cavities, in order to reach larger bandwidth enhancements. It will also be possible to determine which absolute feedback phase leads to the wider chaos bandwidth, to see if it is phase-conjugate feedback that leads to the largest enhancement. Finally, the theoretical results should allow identifying the minimum length of the long cavity required to achieve sufficient chaos bandwidth, which will help the design and realization of an integrated wideband chaos generator.

\section{REFERENCES}

[1] D. Thomson et al., "Roadmap on silicon photonics," J. Opt., vol. 18, no. 7 2016, Art. no. 073003.

[2] L. Vivien and L. Pavesi, Handbook of Silicon Photonics. Boca Raton, FL, USA: CRC Press, Apr. 2013.

[3] G. Roelkens et al., "Silicon-based heterogeneous photonic integrated circuits for the mid-infrared," Opt. Mater. Express, vol. 3, no. 9 , pp. 1523-1536, Sep. 2013.

[4] A. Rickman, "The commercialization of silicon photonics," Nature Photon., vol. 8, no. 8, pp. 579-582, Aug. 2014. 
[5] C. Sun et al., "Single-chip microprocessor that communicates directly using light," Nature, vol. 528, no. 7583, pp. 534-538, Dec. 2015.

[6] M. Asghari and A. V. Krishnamoorthy, "Silicon photonics: Energyefficient communication," Nature Photon., vol. 5, no. 5, pp. 268-270, May 2011.

[7] Y. A. Vlasov, "Silicon CMOS-integrated nano-photonics for computer and data communications beyond 100 g," IEEE Commun. Mag., vol. 50, no. 2, pp. s67-s72, Feb. 2012

[8] M. J. R. Heck, "Grating coupler enabled optical isolators and circulators for photonic integrated circuits," IEEE J. Sel. Topics. Quantum Electron., vol. 21, no. 4, pp. 361-369, Jul. 2015.

[9] A. W. Fang et al., "Electrically pumped hybrid AlGaInAs-silicon evanescent laser," Opt. Express, vol. 14, no. 20, pp. 9203-9210, Oct. 2006.

10] A. W. Fang et al., "Hybrid silicon evanescent devices," Mater. Today, vol. 10, no. 78, pp. 28-35, Jul. 2007.

11] G. Roelkens et al., "III-V/Si photonics by die-to-wafer bonding," Mater Today, vol. 10, no. 78, pp. 36-43, Jul. 2007.

12] H. Park et al., "Device and integration technology for silicon photonic transmitters," IEEE J. Sel. Topics. Quantum Electron., vol. 17, no. 3, pp. 671-688, May 2011.

[13] A. Y. Liu et al., "High performance continuous wave $1.3 \mathrm{~m}$ quantum do lasers on silicon," Appl. Physics Lett., vol. 104, no. 4, Jan. 2014, Art. no. 041104.

[14] S. Chen et al., "Electrically pumped continuous-wave IIIV quantum dot lasers on silicon," Nature Photon., vol. 10, no. 5, pp. 307-311, May 2016.

[15] G. H. Duan et al., "Hybrid IIIV on silicon lasers for photonic integrated circuits on silicon," IEEE J. Sel. Topics. Quantum Electron., vol. 20, no. 4, pp. 158-170, Jul. 2014

16] K. Schires et al., "Dynamics of hybrid III-V silicon semiconductor lasers for integrated photonics," IEEE J. Sel. Topics Quantum Electron., vol. 22 no. 6, pp. 43-49, Nov. 2016.

17] A. Argyris, M. Hamacher, K. E. Chlouverakis, A. Bogris, and D. Syvridis, "Photonic integrated device for chaos applications in communications," Physical Rev. Lett., vol. 100, no. 19, May 2008, Art. no. 194101.

[18] A. Argyris, S. Deligiannidis, E. Pikasis, A. Bogris, and D. Syvridis, "Implementation of $140 \mathrm{~Gb} / \mathrm{s}$ true random bit generator based on a chaotic photonic integrated circuit," Opt. Express, vol. 18, no. 18, pp. 18 763-18 768, Aug. 2010.

[19] R. Takahashi et al., "Fast physical random bit generation with photonic integrated circuits with different external cavity lengths for chaos generation," Opt. Express, vol. 22, no. 10, pp. 11 727-11 740, May 2014.

20] A. K. Dal Bosco et al., "Cycles of self-pulsations in a photonic integrated circuit," Physical Rev. E, vol. 92, no. 6, Dec. 2015, Art. no. 062905.

21] A. K. Dal Bosco et al., "Photonic integrated circuits unveil crisis-induced intermittency," Opt. Express, vol. 24, no. 19, pp. 22 198-22 209, Sep. 2016.

[22] J. Ohtsubo, Semiconductor Lasers: Stability, Instability and Chaos. New York, NY, USA: Springer-Verlag, 2013.

[23] A. Argyris et al., "Chaos-based communications at high bit rates using commercial fibre-optic links," Nature, vol. 438, no. 7066, pp. 343-346, Nov. 2005.

24] H. Someya, I. Oowada, H. Okumura, T. Kida, and A. Uchida, "Synchronization of bandwidth-enhanced chaos in semiconductor lasers with optical feedback and injection," Opt. Express, vol. 17, no. 22, pp. 19 536-19 543, Oct. 2009.

[25] $\tilde{A}$. Mercier, D. Wolfersberger, and M. Sciamanna, "Improving the chaos bandwidth of a semiconductor laser with phase-conjugate feedback," Proc. SPIE, vol. 9892, 2016, Art. no. 98 920G.

[26] M. Zhang et al., "Generation of broadband chaotic laser using dualwavelength optically injected Fabry-Perot laser diode with optical feedback," IEEE Photon. Technol. Lett., vol. 23, no. 24, pp. 1872-1874, Dec. 2011.

27] D. Lenstra, "Relaxation oscillation dynamics in semiconductor diode lasers with optical feedback," IEEE Photon. Technol. Lett., vol. 25, no. 6 , pp. 591-593, Mar. 2013.

28] D. DAgostino, H. P. M. M. Ambrosius, M. K. Smit, and D. Lenstra, "Integrated laser with optical feedback shows suppressed relaxation-oscillation dynamics," IEEE Photon. Technol. Lett., vol. 27, no. 21, pp. 2292-2295, Nov. 2015.

[29] J. Zhao et al., "Stability of a monolithic integrated filtered-feedback laser," Opt. Express, vol. 20, no. 26, pp. B270-B278, Dec. 2012. [Online]. Available: https://www.osapublishing.org/abstract.cfm?uri=oe-20-26-B270

30] O. Brox et al., "High-frequency pulsations in DFB lasers with amplified feedback," IEEE J. Quantum Electron., vol. 39, no. 11, pp. 1381-1387, Nov. 2003.
[31] L. Yu et al., "Monolithically integrated amplified feedback lasers for highquality microwave and broadband chaos generation," J. Lightw. Technol. vol. 32 , no. 20 , pp. 3595-3601, Oct. 2014

[32] D. Tauber and J. E. Bowers, "Dynamics of wide bandwidth semiconductor lasers," Int. J. High Speed Electron. Syst., vol. 8, no. 3, pp. 377-416, Sep. 1997.

[33] B. R. Cemlyn, M. J. Adams, I. D. Henning, and D. Labukhin, "Detuning boundaries of linear and nonlinear dynamics in a system of coupled lasers," IEEE J. Sel. Topics Quantum Electron., vol. 21, no. 6, pp. 514-521, Nov. 2015.

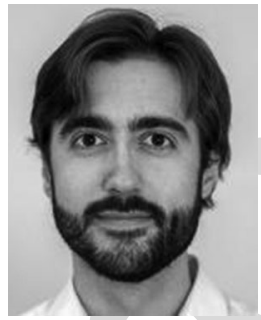

Kevin Schires received the Diplôme d'Ingénieur degree, specializing in signal processing and telecommunications, from the École Supérieure d'Ingénieurs en Électronique et Électrotechnique, Paris, France, and the Ph.D. degree in semiconductor electronics from the University of Essex, Colchester, U.K. He is currently holding a postdoctoral position in the Communications and Electronic Department, Telecom Paristech (former École Nationale Supérieure des Télécommunications) focused on the study of the dynamics of novel semiconductor laser sources under optical injection and optical feedback.

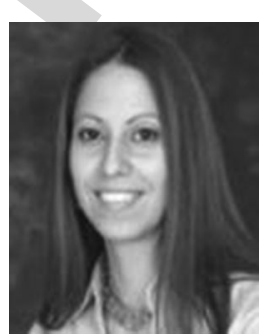

Sandra Gomez received the Bachelor's and Master's degrees in electrical engineering from Stevens Institute of Technology, Hoboken, NJ, USA, with a concentration in digital signal processing (DSP). She is currently the Technical Director of the International Technology Center, France, part of the US Army Research, Development, and Engineering Command (RDECOM) Forward Element Center Atlantic, Paris, France. She previously studied acoustic source localization and the tradeoffs of conventional and adaptive beamforming techniques using different uniform circular array configurations to differentiate between interfering signals and a particular signal of interest. She joined the Ph.D. program at TELECOM Paris Tech in September 2015. Her thesis is focused on advanced laser diodes using new materials in particular those with III-V on silicon, nonlinear dynamics and optical chaos in semiconductor laser systems. She was with the US Army RDECOM Armament Research, Development, and Engineering Center where she served as a Project Officer in the Acoustic and Networked Sensor Division with specific program responsibility in the development of advanced acoustic sensors. She has a strong foundation in systems engineering and extensive experience in defense acquisition, program management, and life cycle support.

Antonin Gallet received the Master's degree of science from Ecole Normale suprieure de Cachan, Cachan. France, and the Master's degree of engineering from Institut doptique, Palaiseau, France, in 2015. He started the Ph.D. research with III-V Lab, Palaiseau, France, and Télécom Paristech, Paris, France, in December 2015 to work on III-V/SOI lasers. 
Guang-Hua Duan (S'88-M'90-SM'01) received the Doctorate degree in applied physics from the École Nationale Supérieure des Télécommunications (Telecom ParisTech), Paris, France, in 1991. He was habilitated to direct researches by Université de ParisSud in 1995 . He is currently the Leader of the research team Silicon Photonics within III-V Lab, which is a joint laboratory of Nokia, Thales, and CEA Leti, and also the Head of the Research Department Heterogeneous integration of III-V on silicon within Nokia Bell Labs. He is also a Guest Professor in Ecole Supérieure dÉlecricité, and École Supérieure dOptique, giving lectures in the fields of electromagnetism, optoelectronics, and laser physics. Previously, he was an Assistant, then an Associate Professor at Télécom-ParisTech from 1992 to 2000. He was with the University of Maryland as a Visiting Associate Professor from 1998 to 1999. He joined Opto+, Alcatel Research \& Innovation Center in Marcoussis in October 2000. He is an author or coauthor of more than 100 journal papers, 250 conference papers, 30 patents, and a contributor to 4 book chapters.

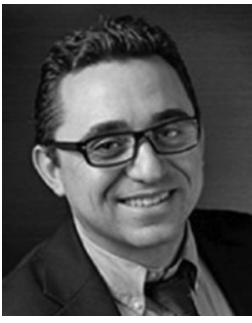

Frédéric Grillot was born in Versailles, France, on 635 August 22, 1974. He received the M.Sc. degree from 636 the University of Dijon, Dijon, France, in 1999, and 637 the Ph.D. degree from the University of Besançon, 638 Besançon, France, in 2003. His doctoral research ac- 639 tivities were conducted within the Optical Compo- 640 nent Research Department, Alcatel-Lucent, working 641 on the effects of the optical feedback in semiconduc- 642 tor lasers, and the impact this phenomenon has on 643 optical communication systems. From 2003 to 2004, 644 he was with the Institut dÉlectronique Fondamentale 645 (University Paris-Sud) where he focused on integrated optics modeling and on 646 Si-based passive devices for optical interconnects. From September 2004 to 647 September 2012, he was with the Institut National des Sciences Appliquées as 648 an Assistant Professor. From 2008 to 2009, he was also a Visiting Professor at the 649 University of New-Mexico, Albuquerque, NM, USA, leading research in opto- 650 electronics at the Center for High Technology Materials. Since October 2012, he 651 has been working with Telecom Paristech (alias École Nationale Supérieure des 652 Télécommunications), Paris, France, where he became an Associate Professor 653 then a Full Professor in January 2017. Since August 2015, he has also been serv- 654 ing as a Research Professor at the University of New-Mexico. He is the author 655 or coauthor of 77 journal papers, 1 book, 3 book chapters, and more than 170656 contributions in international conferences and workshops. His current research 657 interests include advanced quantum confined devices using new materials such 658 as quantum dots and dashes, light emitters based on intersubband transitions, 659 nonlinear dynamics and optical chaos in semiconductor lasers systems, as well 660 as microwave and silicon photonics applications including photonic clocks and 661 photonic analog-to-digital converters. He is an Associate Editor for Optics Ex- 662 press, a Senior Member of the SPIE and the IEEE Photonics Society, as well as 663 a Member of the OSA. 
666

NOTICES

667

668

669

670

671

672

673

674

675

676

677

678

679

680

681
- Author: If you have not completed your electronic copyright form (ECF) and payment option please return to Scholar One "Transfer Center." In the Transfer Center you will click on "Manuscripts with Decisions" link. You will see your article details and under the "Actions" column click "Transfer Copyright." From the ECF it will direct you to the payment portal to select your payment options and then return to ECF for copyright submission.

- Author: Please be aware that authors are required to pay overlength page charges $\$ 220$ per page if the paper is longer than 8 pages for Contributions and +12 pages for Invited Papers. If you cannot pay any or all of these charges please let us know.

- Author: This pdf contains 2 proofs. The first half is the version that will appear on Xplore. The second half is the version that will appear in print. If you have any figures to print in color, they will be in color in both proofs.

- Author: The option to publish your paper as Open Access expires when the paper is fully published on Xplore. Only papers published in "Early Access" may be changed to Open Access.

QUERIES

Q1. Author: If you have not completed your electronic copyright form (ECF) and payment option please return to Scholar One "Transfer Center". In the Transfer Center you will click on "Manuscripts with Decisions" link. You will see your article details and under the "Actions" column click "Transfer Copyright". From the ECF it will direct you to the payment portal to select your payment options and then return to ECF for copyright submission. 


\title{
Passive Chaos Bandwidth Enhancement Under Dual-Optical Feedback with Hybrid III-V/Si DFB Laser
}

\author{
Kevin Schires, Sandra Gomez, Antonin Gallet, Guang-Hua Duan, Senior Member, IEEE, and Frédéric Grillot
}

\begin{abstract}
The chaotic dynamics of a DFB laser are studied experimentally under a combination of short and long feedbacks. Chaos bandwidth enhancement is demonstrated using a hybrid III-V/Si DFB laser with a large relaxation oscillation frequency (ROF) of $14 \mathrm{GHz}$. The impact of short feedback on the ROF is studied and an increase of $2 \mathbf{~ G H z}$ is observed. Under long feedback, the route to chaos of the device and its dependence on the short feedback dynamics are studied. The short feedback allows tuning the chaotic dynamics obtained under long feedback, and the increase of the ROF translates into an enhancement of the chaos bandwidth to above $16 \mathrm{GHz}$. This configuration can allow generation of wideband chaos using a single laser source in a photonic integrated circuit.
\end{abstract}

Index Terms-III-V materials, nonlinear dynamics, optical feedback, silicon photonics, secure communications.

\section{INTRODUCTION}

$\mathbf{S}$ ILICON photonics offer tight integration of a variety of active and passive optical and electrical components, and gained so much interest in the last decade that it is now considered one of the most promising technology for optical applications [1], [2]. Building on the mature fabrication techniques first developed for microelectronics allows creating photonic integrated circuits (PICs) with a high density of optical components, in high volumes and at low costs. Academic and industrial efforts led to the development of novel technical solutions for a variety of domains including sensing, measurement instrumentation, optical signal processing and telecommunications

Manuscript received February 7, 2017; revised March 30, 2017 and June 29, 2017; accepted June 30, 2017. This work was supported by the European Union Horizon 2020 Programme under the PICs4All Project (http://www.pics4all.jeppix.eu). (Corresponding author: Kevin Schires.)

K. Schires and S. Gomez are with Télécom ParisTech, Université Paris-Saclay, Paris 75634, France (e-mail: schires@enst.fr; sandra.gomez@ telecom-paristech.fr).

A. Gallet is with Télécom ParisTech, Université Paris-Saclay, Paris 75634, France, and also with the III-V Lab, Joint Laboratory of Nokia Bell Labs, Thales Research and Technology, and CEA-Leti, Fresnel 91767, France (e-mail: antonin.gallet@3-5lab.fr).

G.-H. Duan are with the III-V Lab, Joint Laboratory of Nokia Bell Labs, Thales Research and Technology, and CEA-Leti, Fresnel 91767, France (e-mail: guanghua.duan@3-5lab.fr).

F. Grillot is with Télécom ParisTech, Université Paris-Saclay, Paris 75634, France, and also with the Center for High Technology Materials, University of New Mexico, Albuquerque, NM 87106-4343 USA (e-mail: grillot@ telecom-paristech.fr).

Color versions of one or more of the figures in this paper are available online at http://ieeexplore.ieee.org.

Digital Object Identifier 10.1109/JSTQE.2017.2732830
[3], [4]. Recent advances in data centers and informatics [5] reveal how photonic integration will become increasingly used for data transmission, either inside a chip or for short-access and long-haul telecommunications networks [6], [7].

Each discrete component used in electro-optical devices had to be redesigned for integration on $\mathrm{Si}$, and a number of research works allowed developing novel integrated modulators, photodetectors, isolators, polarization controllers, amplifiers, as well as novel laser sources [8]. As laser cavities had to be adapted for integration into PICs, novel fabrication techniques were derived for the growth of the active material, and novel designs of the resonant cavity were proposed [9]-[12]. The active III-V material is generally not grown directly on a Si substrate but rather wafer bonded onto a Si waveguide: lattice mismatches between III-V compounds and $\mathrm{Si}$ as well as different thermal expansion lead to dislocation in Quantum Well (QW) materials and to poor device reliability. Novel Quantum Dot (QD) laser sources grown directly on Si have recently been reported [13], [14]: unlike QW materials, the localization of carriers in QDs make these less sensitive to defects. Complex laser cavities for single and multimode laser sources have been proposed [15], but such designs were shown to suffer from stability issues due to internal feedback sources [16] and a simpler DFB structure is studied in this work.

Multisection lasers have been demonstrated on PICs, and devices with an integrated external feedback cavity for use as chaotic emitters are of interest for both academic and industrial research [17]-[19]. PICs creating an external cavity with a phase section are important for research purposes as they allow study of the behavior of lasers under optical feedback with short cavities of controllable length, thus giving new insights into laser dynamics [20], [21]. Under optical feedback of increasing strength, the laser's relaxation oscillations become excited and lead to chaotic oscillation of the laser output [22]. PICs containing lasers with and external optical feedback cavity are thus very important for practical applications, as they allow the use of chaotic emitters in communication networks without imposing volume constraints for integration into existing emitter and receiver modules.

Two major applications of chaotic emitters in communication networks are random number generation and most importantly secure communications [23]. Chaotic communications rely on hiding data within a broad chaotic spectrum at the emitter level, and the data is recovered in the receiver. The bandwidth of the 
amplified feedback should thus be maximized to increase transmission rates while keeping the data secure, and several schemes were proposed to obtain large chaos bandwidth, defined as the frequency under which $80 \%$ of the RF power is found [24]. Passive enhancement of the chaos bandwidth was proposed using phase-conjugate feedback and a high-reflectivity mirror [25]. Active solutions using optical injection from two laser sources in addition to optical feedback demonstrated chaos bandwidths as high as $32 \mathrm{GHz}$ [26].

Another way of generating broad chaos is to increase the relaxation oscillation frequency (ROF) of the laser source. Under optical feedback, the generated chaotic spectra scale with the relaxation frequency of the laser, as chaotic dynamics build up from enhanced relaxation oscillations. In addition, the ROF can be tuned using optical feedback, depending on the feedback phase and strength [22], [27], [28], and that dynamics can be generated by tailoring the feedback and controlling its phase [29]. It was demonstrated that PICs with an integrated amplified external cavity with a phase section allowed forcing self-pulsations in the laser at a frequency tuneable to above $40 \mathrm{GHz}$ [30], [31].

In this article, we study the enhancement of the bandwidth of chaotic dynamics using a combination of two passive cavities of different scales. Chaos bandwidth enhancement is demonstrated using a hybrid III-V/Si QW DFB laser in order to prove applications to PICs. As the optical cavities are created in a fibered setup and not within the chip, only low feedback strengths are achieved. This study shows for the first time the evolution of the dynamics of a hybrid III-V/Si DFB laser with the strength of a long feedback or the phase of a short feedback, and combinations of the two. In Section I, the device is presented and characterized, and the impact of internal reflections on laser operation is discussed. The free-running laser exhibits high relaxation oscillations of $14 \mathrm{GHz}$ which make it ideal for this study as large ROF simplifies the access to wide chaos bandwidths. The route to chaos of the hybrid laser under long feedback is studied in Section III, and compared with that of a commercial III-V DFB laser, showing similar behaviors between the two types of laser despite significant differences in their structures. In Section IV, the laser is subject to a short feedback cavity which allows increasing its ROF by $2 \mathrm{GHz}$. In Section V, the laser is subject to both feedback cavities, and the chaos bandwidth is found to be enhanced in the same fashion as the ROF. The chaos bandwidth is increased by up to $13 \%$ and reaches a maximum of $16.4 \mathrm{GHz}$, showing that this combination of two feedbacks allows controlling the bandwidth and characteristics of the generated chaos despite the very low feedback strength allowed by the setup. Such passive chaos bandwidth enhancement can be applied to standard III-V DFB lasers, and the setup is transposable to a PIC constituted of the laser and two cavities with optional phase sections, allowing wide chaos generation with low power consumption.

\section{EXPERIMENTAL APPARATUS}

\section{A. Device Studied}

Fig. 1 presents a schema of the DFB structure studied. The $1 \mathrm{~mm}$-long hybrid III-V Silicon-on-Insulator (SOI) device is

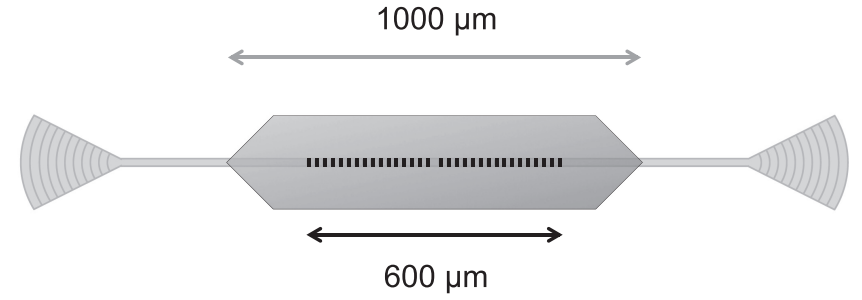

(a)

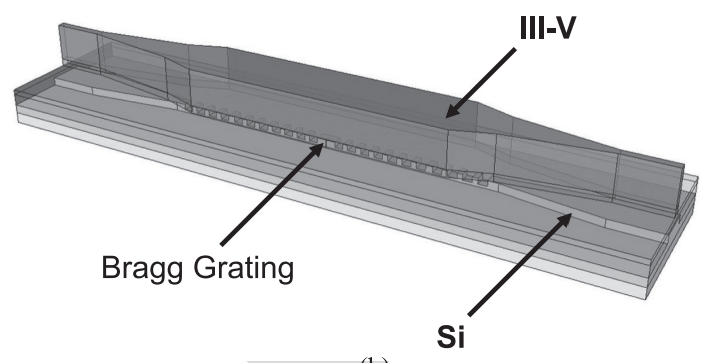

(b)

Fig. 1. (a) Schematic and (b) structure of the device studied, showing the Si waveguide (yellow) and the III-V material (green). The vertical couplers are represented in (a) at both extremities of the $\mathrm{Si}$ waveguide.

fabricated using a III-V QW active medium bonded on top of 132 the processed silicon waveguide, and constituted of a DFB laser 133 with tapers on each side [15]. To ensure single-mode operation, 134 a $50 \mathrm{~nm}$-deep and $600 \mu \mathrm{m}$-long Bragg grating with a quarter- 135 wavelength phase shift in the center is etched on the silicon 136 waveguide. The strength of the grating is chosen such that the 137 product $\kappa L_{\text {bragg }}$ is of a few units, and the period of the grating 138 is of $240 \mathrm{~nm}$. The light is coupled from the $\mathrm{Si}$ waveguide to the 139 III-V material with adiabatic tapers, and outcoupled using Verti- 140 cal Bragg Gratings (VBG) on both side of the device. The VBG 141 couples the light out of the laser with an angle of $80^{\circ}$ from the 142 waveguide, and light was thus coupled vertically using a fiber 143 positioned above the laser, with a $10^{\circ}$ angle from the normal 144 to the lasers surface. The VBG were only necessary for testing 145 purposes, in order to characterize several unprocessed devices 146 on the same bar, and in a PIC they would not be positioned after 147 the laser. They however affected our study in two ways: first via 148 their transmission losses of approximately $7 \mathrm{~dB}$, which will be 149 discussed later, and secondly by their parasitic reflectivity (be- 150 low $-23 \mathrm{~dB}$ ) which affected laser operation when biased high 151 above threshold.

\section{B. Characterisation}

Fig. 2(a) presents the evolution of the power coupled into an anti-reflection (AR)-coated lens-ended fiber with the bias current. All measurements presented in this paper were performed at $20{ }^{\circ} \mathrm{C}$. Around the threshold of about $45 \mathrm{~mA}$, the laser exhibits slight competition between two modes which translates into a kink in the curve. Between 50 and $150 \mathrm{~mA}$, very stable single-mode operation is observed with a side-mode suppression ratio above $50 \mathrm{~dB}$. Above 4 times threshold, the laser exhibited power drops which were also observed on the other lasers of the same bar, but differed from device to device. Such behavior hints that parasitic reflections are present within the devices: the vertical couplers and tapers create reflections which only 


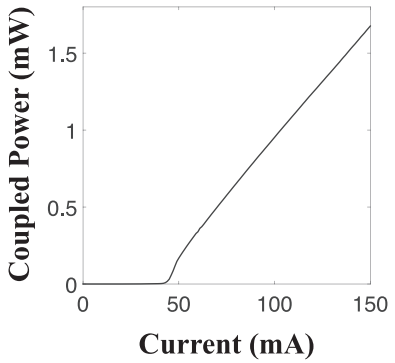

(a)

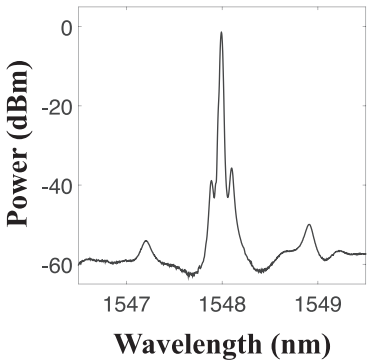

(b)

Fig. 2. (a) Evolution of the power coupled using an AR-coated lensed fiber with the pump current. (b) Optical spectrum at a bias current of $140 \mathrm{~mA}$.

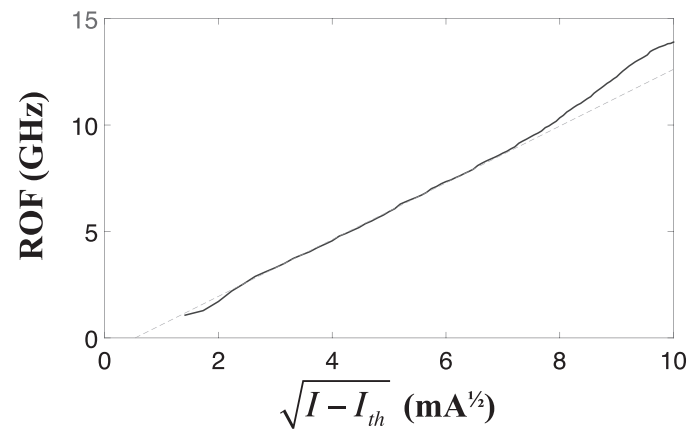

Fig. 3. Evolution of the ROF with the square root of the current overdrive above threshold.

seem to affect the laser far above threshold through variations of the optical power or changes in the ROF, as will be discussed next. As the amount of parasitic reflections varies from device to device, different sorts of power variation would indeed be expected between the different lasers. Fig. 2(b) presents the spectrum of the laser at $140 \mathrm{~mA}$, showing the well-suppressed side-modes as well as sidebands characteristic of relaxation oscillations. These spectra were measured using a Yenista OSA20 optical spectrum analyzer with a $20 \mathrm{pm}$ resolution, and these sidebands could be measured thanks to the rather high value of the ROF.

Fig. 3 presents the evolution of the ROF as a function of the square root of the current overdrive above threshold. It can be noted that very high frequencies are observed as the ROF reaches $14 \mathrm{GHz}$ at $150 \mathrm{~mA}$. Usually, due to gain compression, the power evolves in a sublinear fashion with increasing bias current, and the squared ROF follows the same trend. Here, it can be seen that the ROF rather evolves in the opposite way as it gradually increases above the linear fitting shown as a dashed line.

We believe that these high values of ROF stem from the use of Aluminium in the III-V compound forming the QW [32], but also from the aforementioned internal feedback. Under optical feedback, the ROF can oscillate around its free-running value depending on the feedback strength and delay [22]. Here, the internal feedback conditions thus allow a small enhancement of the ROF for some bias currents, showing an effect of parasitic feedback very different from the detrimental impact it can have further above threshold.

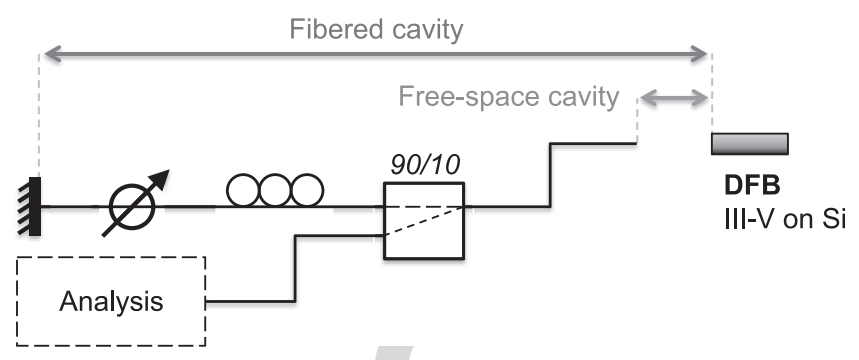

Fig. 4. Experimental setup allowing combination of short and long feedback.

\section{Experimental Setup}

Fig. 4 presents the experimental setup used for the following measurements [16]. The DFB is kept at a bias current of $146 \mathrm{~mA}$ and at $20^{\circ} \mathrm{C}$. The light of the DFB laser is coupled using either an AR-coated lensed fiber or a cleaved uncoated one. The cleaved fiber allows creating a short free-space feedback cavity [33] of the order of $100 \mu \mathrm{m}$, re-injecting about $3 \%$ of the light back into the device with a time delay below 1 ps. On the other hand, the lens-ended fiber minimizes such reflections and only allows light from the setup to be re-injected into the device. In the fiberized setup, a 90/10 splitter is used to create a feedback path consisting of a polarization controller and a Yenista BackReflector (BKR) module, equivalent to a mirror with variable losses. This long fibered cavity measures approximately $7 \mathrm{~m}$ and allows re-injecting at most $8 \%$ of the light into the device. Note that due to the transmission losses of the vertical couplers, a difference must be made between the light re-injected into the component and the light that reaches the laser cavity. The maximum feedback strengths considering the light that reaches the laser cavity are thus of $0.1 \%$ and $0.3 \%$ for the short and long cavities, respectively.

Using either fiber and controlling the attenuation of the long feedback path allows studying the laser into the following four situations: free-running with only parasitic reflections from the setup, under short (free-space) feedback only, under long (fibered) feedback only, and under a combination of both feedbacks. For the short feedback, while the feedback strength is fixed by the refractive index of the fiber, the phase can be tuned using a piezoelectric actuator allowing gradually moving the fiber towards or away from the device. Concerning the long feedback, while the feedback phase can be changed the same way it does not impact laser behavior given the large external cavity length. Feedback strength can however be tuned by changing the attenuation of the BKR.

\section{LONG FEEDBACK}

To assess the potential of such hybrid lasers as chaotic emitters, we first study the route to chaos that the devices follow under long feedback. The cavity used here is too long to represent the external cavity that would be integrated in a PIC, but it allows pushing the laser into chaotic operation where the phase of the long feedback has little impact on the dynamics. This will later allow us to dissociate the effect of the short and long feedbacks when combined together, as we can consider that the feedback phase will only affect the dynamics induced by the 


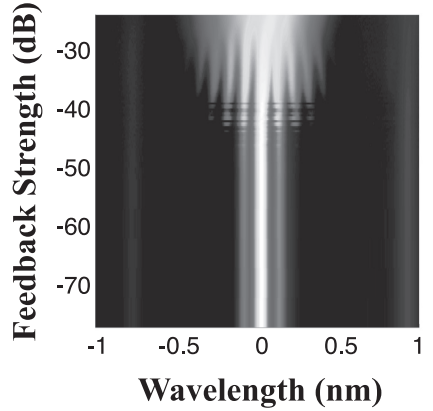

(a) (b)

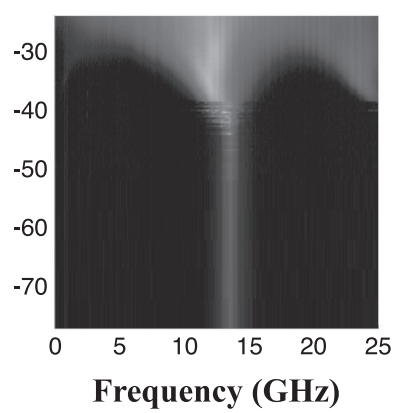

Fig. 5. Evolution of the (a) optical and (b) RF spectra with long feedback strength, using a lens-ended fiber.

short feedback. Fig. 5 presents the evolution of the optical and RF spectra with the long feedback strength, using the lensed fiber. The measurements performed with the lensed fiber have very little dependence on the position of the fiber and thus the feedback phase, as it will be shown later in the case of the freerunning laser. Routes to chaos under long feedback were thus found to be identical for different positions of the fiber.

The route observed is typical of a DFB laser under long optical feedback despite major differences in the device structures: first of all, the hybrid DFB is constituted of two evanescently coupled waveguides, one passive and one active, unlike the standard III-V DFB. In addition, in the hybrid device the tapered regions on each side of the DFB structure may slightly amplify the light fed back into the laser, or even act as source of internal reflections [16]. The proposed chaos bandwidth enhancement method could also be applied to a standard III-V DFB, for which higher feedback strengths could be achieved. Demonstrating the changes in ROF and bandwidth in the case of hybrid lasers is however interesting, as it shows that the complexity of the lasers used in PICs does not necessarily affect their behavior under optical feedback.

For very low feedback strengths, the ROF can be seen at $14 \mathrm{GHz}$ in the RF spectrum. Around $-43 \mathrm{~dB}$ of feedback strength, temporally unstable periodic oscillations start to appear, and stabilize above $-40 \mathrm{~dB}$. These correspond to an excitation of relaxation oscillations that turn into chaotic dynamics above $-30 \mathrm{~dB}$ of feedback strength. Under maximum feedback strength, the bandwidth of the chaotic spectra is of $14.5 \mathrm{GHz}$.

Fig. 6 allows comparing the route to chaos of this hybrid DFB laser with that of a commercial Nokia III-V DFB laser. The laser is operated a three times its threshold where it has a ROF of $8 \mathrm{GHz}$. The routes appear to be very similar. Along the feedback strength axis, the routes appear to be shifted by $6 \mathrm{~dB}$ as the III-V DFB reaches chaotic operation for only $-36 \mathrm{~dB}$ of feedback strength. Note that in the case of the III-V laser, higher feedback strengths were achievable with the same setup as there were no extra losses between the fiber and the laser cavity. It can thus be seen that with the hybrid DFB we are only able to merely enter the chaotic regime, and that much broader spectra could be observed for stronger feedback. Along the wavelength axis, broader spectra are observed for the hybrid DFB in the region of periodic oscillations, as the base frequency of these oscillations

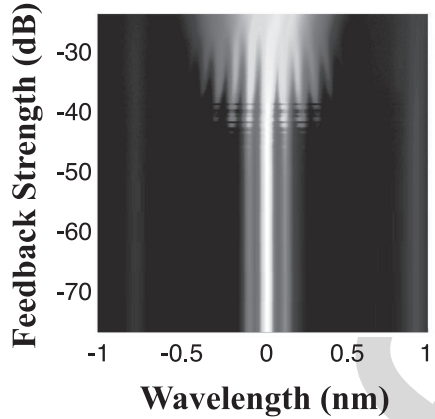

(a)

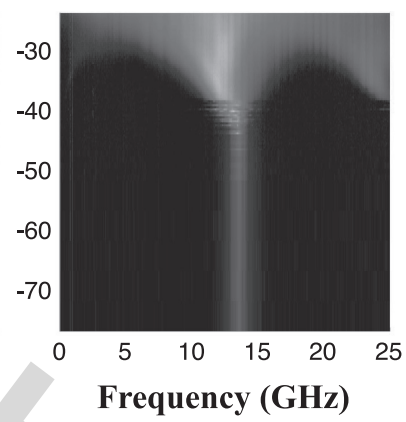

(b)
Fig. 6. Evolution of the optical spectra of a (a) III-V and (b) III-V/Si DFB laser with long feedback strength, using a lens-ended fiber.

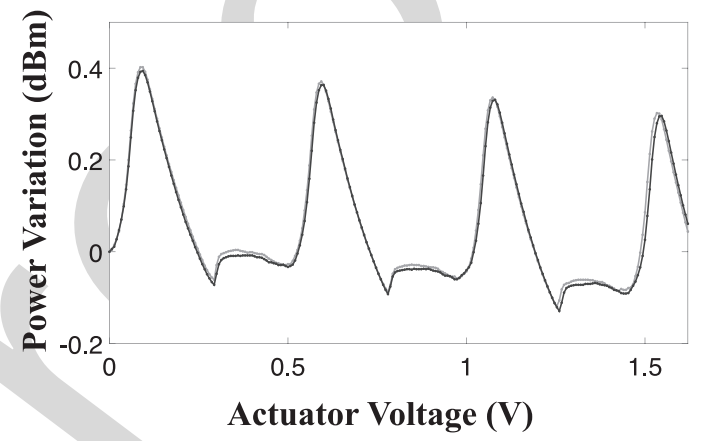

Fig. 7. Variation of the power coupled with the cleaved fiber as a function of the voltage applied to the piezoelectric actuator. Two measurements for different fiber positions are presented in black and grey.

is the ROF, and we expect that the spectra observed for the III-V DFB could be obtained for the hybrid one, broadened by a factor close to the ratio between the two ROFs.

\section{SHORT FEEDBACK}

Using the cleaved fiber and setting the long feedback strength 285 to its minimum of $-79 \mathrm{~dB}$ allows studying the DFB under short 286 feedback only. Fig. 7 presents the variation of the coupled power 287 as a function of the voltage applied to the piezoelectric actuator. 288 As the coupling is only optimized for a voltage of $0 \mathrm{~V}$, the power 289 fades as the fiber is moved away from the device and the figure 290 appears skewed. A clear periodicity can however be observed 291 in the evolution of the power, as we are varying the phase of 292 the short feedback. For convenience, we choose as beginning of 293 each period the main peaks and consider that between each peak, 294 the phase is varied from 0 to $2 \pi$. Note that this does not represent 295 the absolute phase of the feedback, but only a representation 296 of the phase shift within a period.

Such variation of the output power under optical feedback 298 corresponds to the effect of a medium feedback strength and 299 usually exhibits bistability when the mirror is moved one way 300 or the other [22]. Fig. 8 presents a comparison between the 301 evolution of the power and spectrum when the fiber is moved in 302 both directions, as well as reference measurements performed 303 with the lensed fiber. The reference measurements shows the 304 weak impact of all parasitic reflections from the setup, and shows 305 that the cavity created by the cleaved fiber is mainly responsible 306 


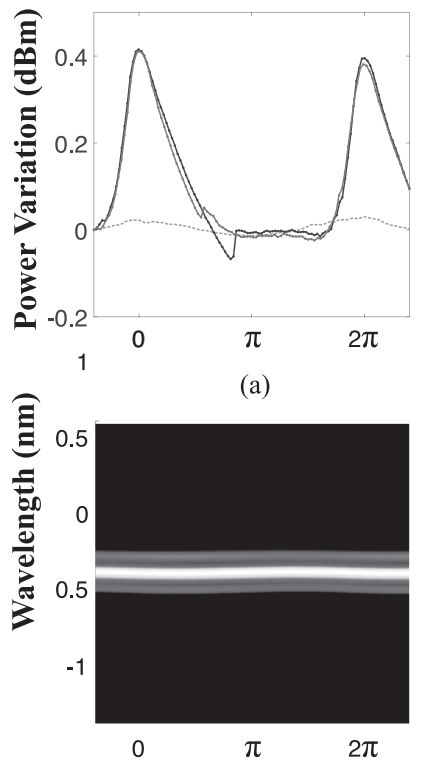

(b)

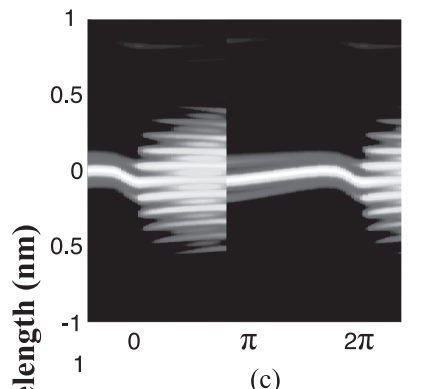

(c)

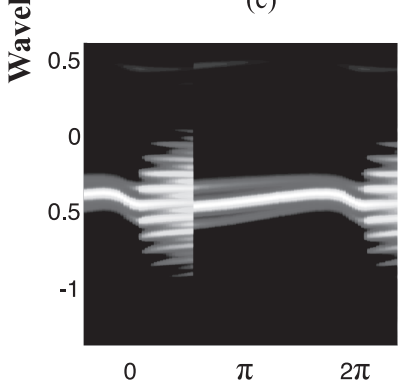

(d)

Feedback Phase (rad)

Fig. 8. (a) Variation of the power coupled with the cleaved fiber within one period when the fiber is moved away (black) or towards (red) the laser. The grey dashed line represents the power coupled when using the lensed fiber. (b) Evolution of the optical spectrum when using the lensed fiber. (c) and (d) Show the evolution of the optical spectrum when moving the fiber away or towards the laser, respectively.

for the power variation presented above. In Fig. 8(a) the gray dashed line shows that the power is oscillating sinusoidally with a low amplitude, which corresponds to the impact of weak feedback. Fig. 8(b) shows that the wavelength varies in a similar fashion. This confirms that any other variation of the power or wavelength is only due to the feedback created by the cleaved fiber. Fig. 8(a) also reveals a slight bistability in the evolution of the power, as the kinks observed after the peak occur for a phase shift of $0.3 \pi$ for one direction and $0.4 \pi$ for the other. While the bistability in the output power is negligible, it makes a significant difference in the optical spectrum. Note that all spectrum maps presented in this work are plotted in $\mathrm{dBm}$, with a logarithmic color scale.

Fig. 8(c) reveals that during each periods, the laser exhibits periodic dynamics between phases of 0 and $0.4 \pi$, with period doubling above $0.2 \pi$. When the kink occurs in the optical power, the laser suddenly stabilises, and it can be seen from the sidebands that the ROF changes with the phase. Fig. 8(d) shows that the same behaviour is observed when the fiber is moved in the opposite direction, thus leading to a wider region of stability. This wider region is of importance for this study as it appears that the largest ROFs are achieved within the bistablity window when the laser is stable, as this is where the mode's sidebands appear to be the farthest apart.

Fig. 9 shows the evolution of the optical and RF spectra of the DFB as the fiber is moved towards the laser, at a shorter distance. In order to clearly see the peak of the relaxation oscillations in the RF spectra, an EDFA set to a fixed output power of $10 \mathrm{dBm}$ was used to amplify the light before detection. A photodetector with a bandwidth of $30 \mathrm{GHz}$ and a Rohde \& Schwartz FSP

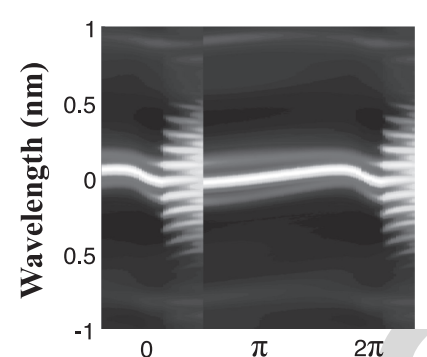

(a)

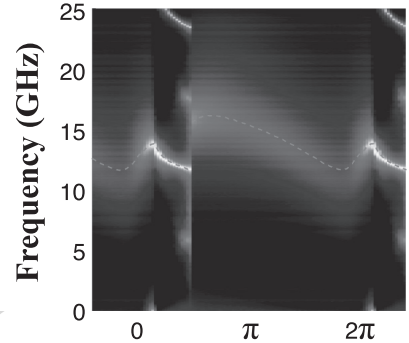

(b)
Feedback Phase (rad)

Fig. 9. Evolution of the (a) optical and (b) RF spectra within one period. The green dashed line shows the evolution of the ROF.

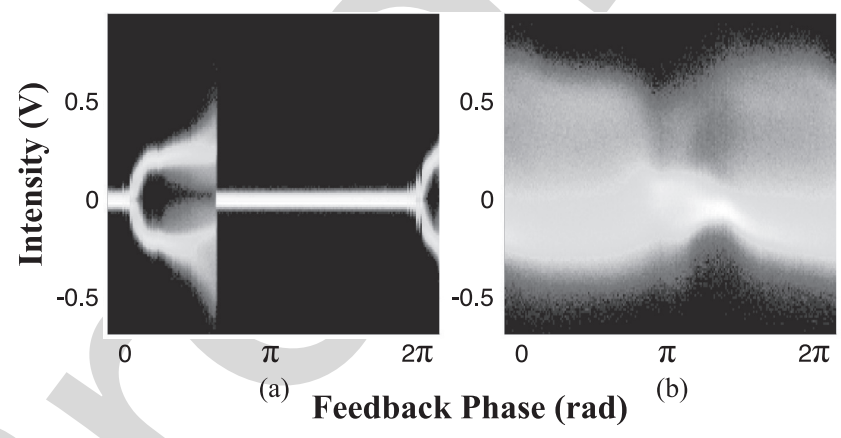

Fig. 10. Bifurcation diagrams as a function of the short feedback phase under (a) minimum and (b) maximum long feedback strength.

$40 \mathrm{GHz}$ electrical spectrum analyzer were used to perform the spectrum measurements. A slightly larger stability region is obtained, and the RF spectra show that within one period, the ROF varies between a minimum of $11 \mathrm{GHz}$ and a maximum of $16 \mathrm{GHz}$, thus allowing reaching frequencies $2 \mathrm{GHz}$ higher than the free-running ROF.

\section{COMbination of FeEdBacks}

Under long feedback only, the hybrid DFB exhibits a classic route to chaos where chaotic dynamics are obtained from the excitation of relaxation oscillations. Under short feedback only, it is possible to tune the value of the ROF while keeping the laser into stable operation. Combining both feedbacks will allow us to study whether the enhancement of the ROF under short feedback can be used to generate broader chaos by first tailoring the ROF and then exciting relaxation oscillations into chaotic ones. In this section, the laser is thus subjected to a combination of short and long feedbacks by using the cleaved fiber and varying the long feedback strength.

Fig. 10 presents the bifurcation diagrams of the dynamics as a function of the phase of the short feedback, under minimum and maximum long feedback strengths. Under minimum long feedback strength, the diagram clearly shows the apparition of the periodic oscillations and the sudden transition from oscillating to stable operation. Under maximum feedback, it can be seen that the chaotic signal changes significantly with the short feedback phase.

Fig. 11 presents the evolution of optical and RF spectra with the position of the cleaved fiber for four levels of long feedback 


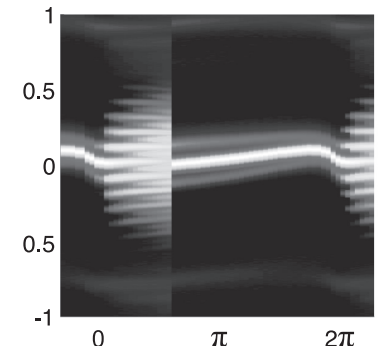

(a)

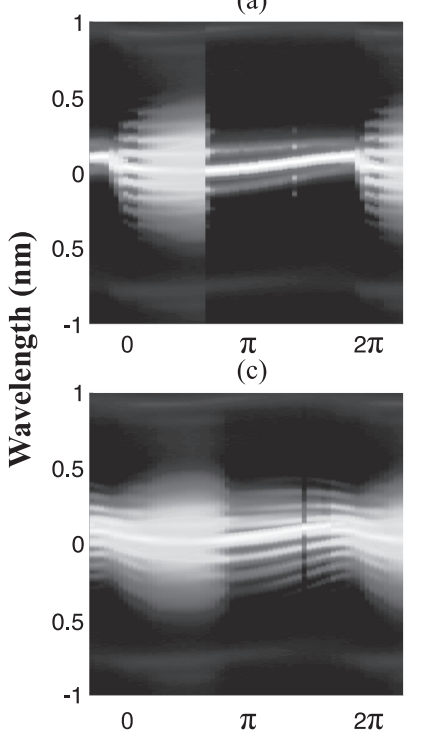

(e)

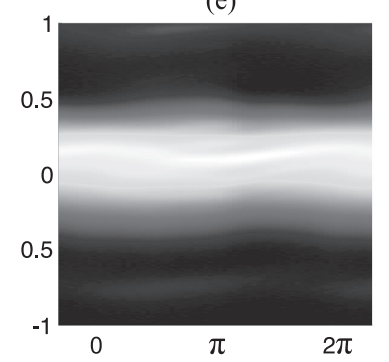

(g)

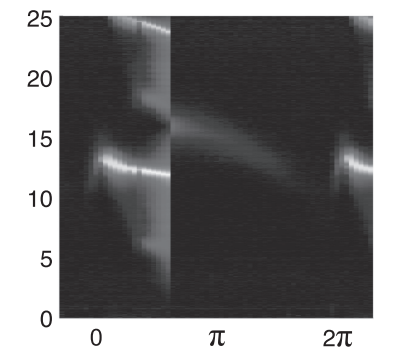

(b)

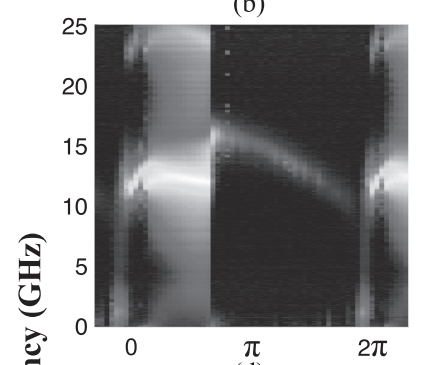

(d)

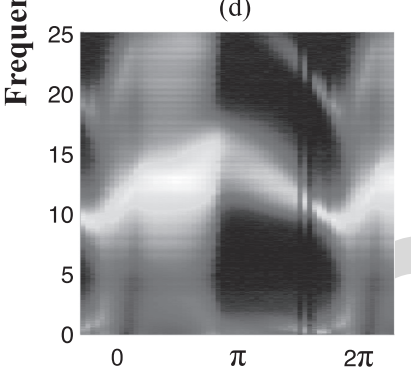

(f)

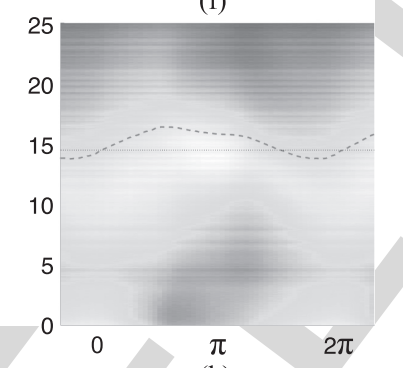

(h)

Fig. 11. Evolution of optical (left) and RF (right) spectra with the position of the cleaved fiber for long feedback strengths of (a), (b) $-79 \mathrm{~dB}$, (c), (d) $-42 \mathrm{~dB}$, (e), (f) $-36 \mathrm{~dB}$ and $(\mathrm{g}),(\mathrm{h})-26 \mathrm{~dB}$. In (h) the black line shows the chaos bandwidth under long feedback only, and the green dashed line shows the evolution of chaos bandwidth with the phase of the short feedback.

strength: the minimum of $-79 \mathrm{~dB},-42 \mathrm{~dB},-36 \mathrm{~dB}$ and the maximum of $-26 \mathrm{~dB}$. No optical amplification was used for the RF spectrum measurements. The route towards chaos under long feedback clearly changes with the short feedback phase, as periodic dynamics appear for different long feedback strengths at the different positions of the cleaved fiber. Regions where periodic oscillations occur under short feedback alone appear to enter chaotic operation first. The sharp transition between oscillating and stable operation gradually disappears as the long feedback strength is increased. In a similar way as in Figs. 5 and 6 , some points exhibit temporally unstable dynamics for feedback phases between $1.3 \pi$ and $1.5 \pi$ and feedback strengths of -42 and -36 , which can be seen as a disappearance of the

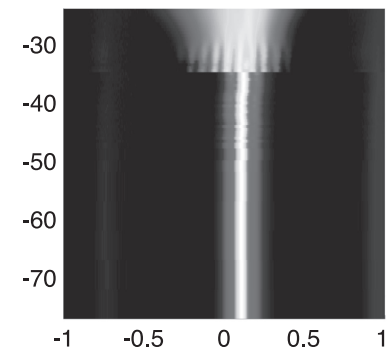

(a)

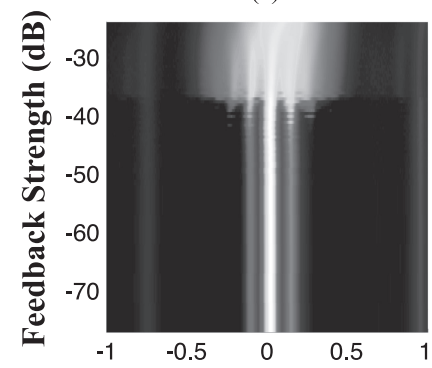

(c)

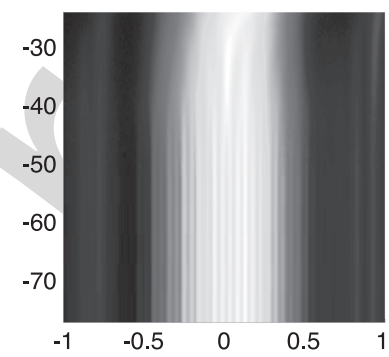

(e)

Wavelength (nm)

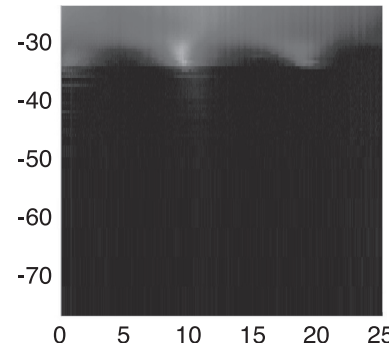

(b)

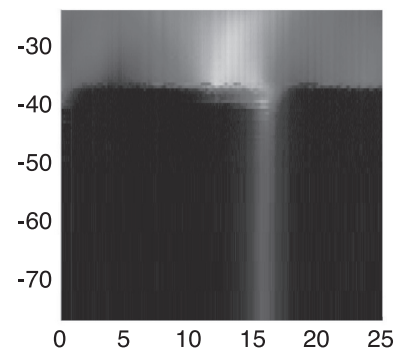

(d)

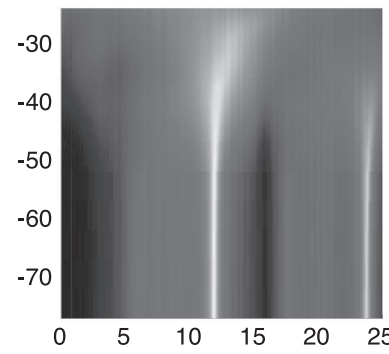

(f)

Frequency (GHz)
Fig. 12. Evolution of the optical (left) and RF (right) spectra with the long feedback strength for three positions of the cleaved fiber. (a) and (b) correspond to a feedback phase of $1.9 \pi$ (minimum ROF, stable). (c) and (d) correspond to a feedback phase of $0.3 \pi$ (maximum ROF, stable). (e) and (f) correspond to a feedback phase of $0.25 \pi$ (strongest oscillations).

dynamics for some isolated feedback phases. It is interesting 378 to observe that, as the long feedback strength is increased, the 379 wavelength of the spectrum peak oscillates in a more and more 380 sinusoidal fashion, such that no bistability was observed under 381 maximum long feedback strength.

The long feedback strength necessary to reach chaotic operation seems to depend on the short feedback phase, and it can be seen in Fig. 11(g) and (h) that the width of the chaotic spectrum varies with the feedback phase too. In Fig. 11(h), the superimposed green dashed line shows the evolution of the chaos bandwidth with the short feedback phase. The black dotted line shows as a reference the bandwidth of the chaos measured under maximum long feedback only, corresponding to the spectrum under maximum long feedback strength in Fig. 5(b). With the addition of short feedback, the chaos bandwidth oscillates between 13.7 and $16.4 \mathrm{GHz}$ by following very closely the evolution of the ROF in Fig. 9(b). Minimum and maximum chaos bandwidth are indeed respectively found close to the feedback phases where minimum and maximum ROF are observed in the absence of long feedback. 


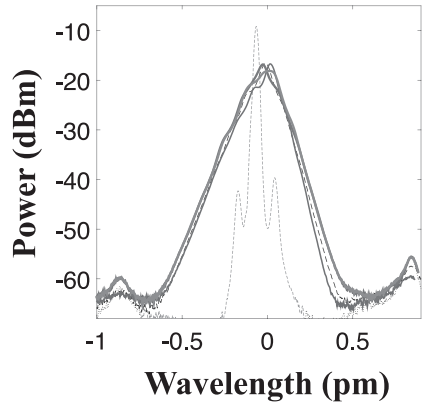

(a)

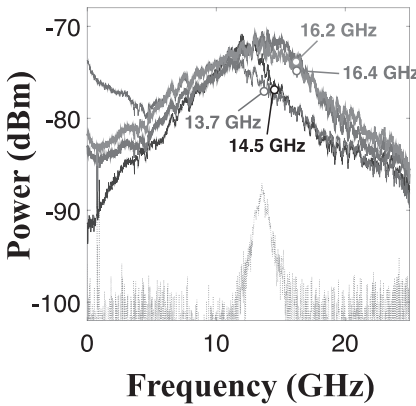

(b)
Fig. 13. Optical (left) and RF (right) spectra under maximum long feedback and their bandwidth, the dashed lines showing the free-running spectra as reference. The black curve corresponds to maximum long feedback alone. The blue (resp. red) curve corresponds to both feedbacks with minimum (maximum) ROF. The green curve corresponds to both feedback with strongest periodic oscillations.

Three specific routes to chaos are thus of interest: those where short feedback does not destabilize the laser but induces either a reduction of the ROF to its minimum value of $11 \mathrm{GHz}$ (phase of $1.9 \pi$ ) or an increase to its maximum value of $16 \mathrm{GHz}$ (phase of $0.3 \pi$ ), and one where short feedback induces the strongest periodic oscillations (phase of $0.25 \pi$ ). Fig. 12 presents these three routes. In the first two routes, the laser remains stable until slightly higher feedback strengths than compared to Fig. 5(a). The window where periodic oscillations are observed is however very narrow, as chaos appears around the same feedback strength of about $-30 \mathrm{~dB}$. In the last route, chaotic dynamics seem to appear for a lower long feedback strength of $-38 \mathrm{~dB}$, but under maximum long feedback the end of the route appears similar to that in Fig. 12(d). This is however not surprising given that the last two routes are measured for short feedback phases that are rather close, and that no discontinuity can be seen in Fig. 11(g) and (h) between these phases.

The optical and RF spectra obtained under maximum long feedback for these three operating conditions are shown in Fig. 13, along with the free-running spectra and the chaotic ones obtained under maximum long feedback alone. Fig. 13(b) shows that the chaotic spectrum obtained for a feedback phase of $1.9 \pi$ leads to the minimum bandwidth of $13.7 \mathrm{GHz}$ observed in Fig. 11(h). Both the other routes lead to a bandwidth of $16.2 \mathrm{GHz}$, despite slight differences in the chaotic spectra.

\section{CONCLUSION}

A combination of long and short feedback are used to respectively generate chaotic dynamics in a DFB laser and tune the chaos bandwidth. The dynamics of a hybrid III-V/Si DFB laser were studied under such combination of feedbacks to show the possibility to achieve passive chaos bandwidth enhancement in PICs. The routes of the dynamics are extensively characterized by varying the phase of the short feedback and strength of the long one. Owing to the large ROF of the free-running laser, chaotic dynamics with a bandwidth of $14.5 \mathrm{GHz}$ can be generated using a long external feedback cavity. The route to chaos appears to be very similar to that of a standard commercial III-V DFBs, but due to the transmission losses of the device's vertical couplers, maximum feedback strengths of only $0.3 \%$ were achievable. The impact of a short external cavity on the laser dynamics revealed a variation of the ROF of $5 \mathrm{GHz}$, with a maximum value of $16 \mathrm{GHz}$. When combining short and long feedbacks, this $2 \mathrm{GHz}$ increase of the ROF translates into a similar increase of the chaos bandwidth, which reaches $16.4 \mathrm{GHz}$.

The tuneability of the chaotic dynamics and of their bandwidth is of interest for applications requiring random number generation, or broad chaos generation. With feedback cavities integrated into a $\mathrm{Si}$ waveguide along with a phase section, higher feedback strengths would be achievable for both short and long feedbacks. Chaotic spectra much wider than those reported in this work could thus be obtained using a single component and passive optical cavities with optional phase sections. This would be of prime importance for the development of low-consumption integrated chaotic transmitters and receivers for secure communications.

As the wide bandwidth relies primarily on the large ROF of the laser, the design of the device is extremely important, and for these applications QW sources may be more appealing than QD sources as QW lasers generally exhibit much higher ROFs than QD ones. In the case of application in a Si PIC, the design of the Si waveguides would be very important too, as all the possible sources of reflections within the PIC will affect the dynamics of the laser. In this work, at least two feedback cavities allow pushing the ROF towards the values reported here: the cavity created by the cleaved fiber, but also the internal parasitic sources of feedback which seem to increase the ROF of the free-running laser already. If sources of feedback are inevitable in a PIC, they can thus be designed to potentially enhance the laser's operation instead of hindering it.

Future work will focus on the simulation of single mode III-V and III-V/Si lasers subject to two external cavities in order to attempt to replicate these experimental results. Conditions to maximize the increase of the ROF under short feedback will thus be studied in the presence of several short cavities, in order to reach larger bandwidth enhancements. It will also be possible to determine which absolute feedback phase leads to the wider chaos bandwidth, to see if it is phase-conjugate feedback that leads to the largest enhancement. Finally, the theoretical results should allow identifying the minimum length of the long cavity required to achieve sufficient chaos bandwidth, which will help the design and realization of an integrated wideband chaos generator.

\section{REFERENCES}

[1] D. Thomson et al., "Roadmap on silicon photonics," J. Opt., vol. 18, no. 7, 2016, Art. no. 073003.

[2] L. Vivien and L. Pavesi, Handbook of Silicon Photonics. Boca Raton, FL, USA: CRC Press, Apr. 2013.

[3] G. Roelkens et al., "Silicon-based heterogeneous photonic integrated circuits for the mid-infrared," Opt. Mater. Express, vol. 3, no. 9 pp. 1523-1536, Sep. 2013.

[4] A. Rickman, "The commercialization of silicon photonics," Nature Photon., vol. 8, no. 8, pp. 579-582, Aug. 2014. 
[5] C. Sun et al., "Single-chip microprocessor that communicates directly using light," Nature, vol. 528, no. 7583, pp. 534-538, Dec. 2015.

[6] M. Asghari and A. V. Krishnamoorthy, "Silicon photonics: Energyefficient communication," Nature Photon., vol. 5, no. 5, pp. 268-270, May 2011.

[7] Y. A. Vlasov, "Silicon CMOS-integrated nano-photonics for computer and data communications beyond 100 g," IEEE Commun. Mag., vol. 50, no. 2, pp. s67-s72, Feb. 2012

[8] M. J. R. Heck, "Grating coupler enabled optical isolators and circulators for photonic integrated circuits," IEEE J. Sel. Topics. Quantum Electron., vol. 21, no. 4, pp. 361-369, Jul. 2015.

[9] A. W. Fang et al., "Electrically pumped hybrid AlGaInAs-silicon evanescent laser," Opt. Express, vol. 14, no. 20, pp. 9203-9210, Oct. 2006.

10] A. W. Fang et al., "Hybrid silicon evanescent devices," Mater. Today, vol. 10, no. 78, pp. 28-35, Jul. 2007.

11] G. Roelkens et al., "III-V/Si photonics by die-to-wafer bonding," Mater Today, vol. 10, no. 78, pp. 36-43, Jul. 2007.

12] H. Park et al., "Device and integration technology for silicon photonic transmitters," IEEE J. Sel. Topics. Quantum Electron., vol. 17, no. 3, pp. 671-688, May 2011.

[13] A. Y. Liu et al., "High performance continuous wave $1.3 \mathrm{~m}$ quantum do lasers on silicon," Appl. Physics Lett., vol. 104, no. 4, Jan. 2014, Art. no. 041104.

[14] S. Chen et al., "Electrically pumped continuous-wave IIIV quantum dot lasers on silicon," Nature Photon., vol. 10, no. 5, pp. 307-311, May 2016.

[15] G. H. Duan et al., "Hybrid IIIV on silicon lasers for photonic integrated circuits on silicon," IEEE J. Sel. Topics. Quantum Electron., vol. 20, no. 4, pp. 158-170, Jul. 2014

16] K. Schires et al., "Dynamics of hybrid III-V silicon semiconductor lasers for integrated photonics," IEEE J. Sel. Topics Quantum Electron., vol. 22, no. 6, pp. 43-49, Nov. 2016

17] A. Argyris, M. Hamacher, K. E. Chlouverakis, A. Bogris, and D. Syvridis, "Photonic integrated device for chaos applications in communications," Physical Rev. Lett., vol. 100, no. 19, May 2008, Art. no. 194101.

[18] A. Argyris, S. Deligiannidis, E. Pikasis, A. Bogris, and D. Syvridis, "Implementation of $140 \mathrm{~Gb} / \mathrm{s}$ true random bit generator based on a chaotic photonic integrated circuit," Opt. Express, vol. 18, no. 18, pp. 18 763-18 768, Aug. 2010.

[19] R. Takahashi et al., "Fast physical random bit generation with photonic integrated circuits with different external cavity lengths for chaos generation," Opt. Express, vol. 22, no. 10, pp. 11 727-11 740, May 2014.

[20] A. K. Dal Bosco et al., "Cycles of self-pulsations in a photonic integrated circuit," Physical Rev. E, vol. 92, no. 6, Dec. 2015, Art. no. 062905.

21] A. K. Dal Bosco et al., "Photonic integrated circuits unveil crisis-induced intermittency," Opt. Express, vol. 24, no. 19, pp. 22 198-22 209, Sep. 2016.

22] J. Ohtsubo, Semiconductor Lasers: Stability, Instability and Chaos. New York, NY, USA: Springer-Verlag, 2013.

[23] A. Argyris et al., "Chaos-based communications at high bit rates using commercial fibre-optic links," Nature, vol. 438, no. 7066, pp. 343-346, Nov. 2005.

24] H. Someya, I. Oowada, H. Okumura, T. Kida, and A. Uchida, "Synchronization of bandwidth-enhanced chaos in semiconductor lasers with optical feedback and injection," Opt. Express, vol. 17, no. 22, pp. 19 536-19 543, Oct. 2009.

[25] $\tilde{A}$. Mercier, D. Wolfersberger, and M. Sciamanna, "Improving the chaos bandwidth of a semiconductor laser with phase-conjugate feedback," Proc. SPIE, vol. 9892, 2016, Art. no. 98 920G.

[26] M. Zhang et al., "Generation of broadband chaotic laser using dualwavelength optically injected Fabry-Perot laser diode with optical feedback," IEEE Photon. Technol. Lett., vol. 23, no. 24, pp. 1872-1874, Dec. 2011.

[27] D. Lenstra, "Relaxation oscillation dynamics in semiconductor diode lasers with optical feedback," IEEE Photon. Technol. Lett., vol. 25, no. 6 , pp. 591-593, Mar. 2013.

28] D. DAgostino, H. P. M. M. Ambrosius, M. K. Smit, and D. Lenstra, "Integrated laser with optical feedback shows suppressed relaxation-oscillation dynamics," IEEE Photon. Technol. Lett., vol. 27, no. 21, pp. 2292-2295, Nov. 2015.

29] J. Zhao et al., "Stability of a monolithic integrated filtered-feedback laser," Opt. Express, vol. 20, no. 26, pp. B270-B278, Dec. 2012. [Online]. Available: https://www.osapublishing.org/abstract.cfm?uri=oe-20-26-B270

30] O. Brox et al., "High-frequency pulsations in DFB lasers with amplified feedback," IEEE J. Quantum Electron., vol. 39, no. 11, pp. 1381-1387, Nov. 2003.
[31] L. Yu et al., "Monolithically integrated amplified feedback lasers for highquality microwave and broadband chaos generation," J. Lightw. Technol. vol. 32 , no. 20 , pp. 3595-3601, Oct. 2014.

[32] D. Tauber and J. E. Bowers, "Dynamics of wide bandwidth semiconductor lasers," Int. J. High Speed Electron. Syst., vol. 8, no. 3, pp. 377-416, Sep. 1997.

[33] B. R. Cemlyn, M. J. Adams, I. D. Henning, and D. Labukhin, "Detuning boundaries of linear and nonlinear dynamics in a system of coupled lasers," IEEE J. Sel. Topics Quantum Electron., vol. 21, no. 6, pp. 514-521, Nov. 2015.

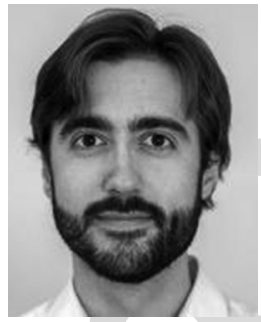

Kevin Schires received the Diplôme d'Ingénieur degree, specializing in signal processing and telecommunications, from the École Supérieure d'Ingénieurs en Électronique et Électrotechnique, Paris, France, and the Ph.D. degree in semiconductor electronics from the University of Essex, Colchester, U.K. He is currently holding a postdoctoral position in the Communications and Electronic Department, Telecom Paristech (former École Nationale Supérieure des Télécommunications) focused on the study of the dynamics of novel semiconductor laser sources under optical injection and optical feedback.

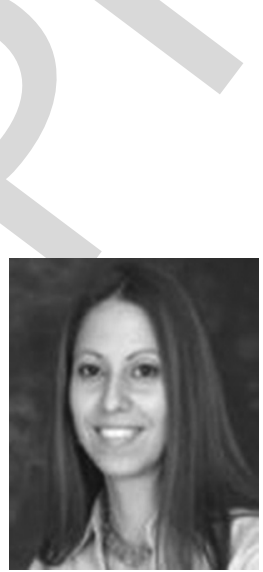

Sandra Gomez received the Bachelor's and Master's degrees in electrical engineering from Stevens Institute of Technology, Hoboken, NJ, USA, with a concentration in digital signal processing (DSP). She is currently the Technical Director of the International Technology Center, France, part of the US Army Research, Development, and Engineering Command (RDECOM) Forward Element Center Atlantic, Paris, France. She previously studied acoustic source localization and the tradeoffs of conventional and adaptive beamforming techniques using different uniform circular array configurations to differentiate between interfering signals and a particular signal of interest. She joined the Ph.D. program at TELECOM Paris Tech in September 2015. Her thesis is focused on advanced laser diodes using new materials in particular those with III-V on silicon, nonlinear dynamics and optical chaos in semiconductor laser systems. She was with the US Army RDECOM Armament Research, Development, and Engineering Center where she served as a Project Officer in the Acoustic and Networked Sensor Division with specific program responsibility in the development of advanced acoustic sensors. She has a strong foundation in systems engineering and extensive experience in defense acquisition, program management, and life cycle support.

Antonin Gallet received the Master's degree of science from Ecole Normale suprieure de Cachan, Cachan. France, and the Master's degree of engineering from Institut doptique, Palaiseau, France, in 2015. He started the Ph.D. research with III-V Lab, Palaiseau, France, and Télécom Paristech, Paris, France, in December 2015 to work on III-V/SOI lasers. 


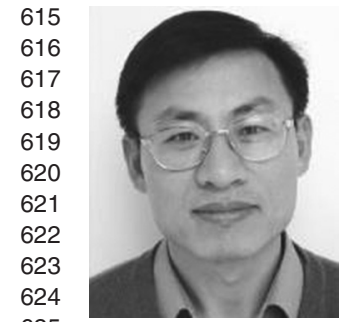

Guang-Hua Duan (S'88-M'90-SM'01) received the Doctorate degree in applied physics from the École Nationale Supérieure des Télécommunications (Telecom ParisTech), Paris, France, in 1991. He was habilitated to direct researches by Université de ParisSud in 1995. He is currently the Leader of the research team Silicon Photonics within III-V Lab, which is a joint laboratory of Nokia, Thales, and CEA Leti, and also the Head of the Research Department Heterogeneous integration of III-V on silicon within Nokia Bell Labs. He is also a Guest Professor in Ecole Supérieure dÉlecricité, and École Supérieure dOptique, giving lectures in the fields of electromagnetism, optoelectronics, and laser physics. Previously, he was an Assistant, then an Associate Professor at Télécom-ParisTech from 1992 to 2000. He was with the University of Maryland as a Visiting Associate Professor from 1998 to 1999. He joined Opto+, Alcatel Research \& Innovation Center in Marcoussis in October 2000. He is an author or coauthor of more than 100 journal papers, 250 conference papers, 30 patents, and a contributor to 4 book chapters.

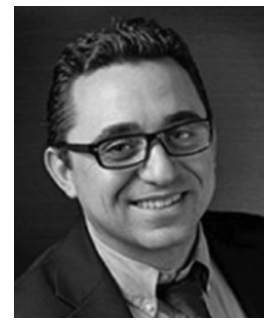

Frédéric Grillot was born in Versailles, France, on 635 August 22, 1974. He received the M.Sc. degree from 636 the University of Dijon, Dijon, France, in 1999, and 637 the Ph.D. degree from the University of Besançon, 638 Besançon, France, in 2003. His doctoral research ac- 639 tivities were conducted within the Optical Compo- 640 nent Research Department, Alcatel-Lucent, working 641 on the effects of the optical feedback in semiconduc- 642 tor lasers, and the impact this phenomenon has on 643 optical communication systems. From 2003 to 2004, 644 he was with the Institut dÉlectronique Fondamentale 645 (University Paris-Sud) where he focused on integrated optics modeling and on 646 Si-based passive devices for optical interconnects. From September 2004 to 647 September 2012, he was with the Institut National des Sciences Appliquées as 648 an Assistant Professor. From 2008 to 2009, he was also a Visiting Professor at the 649 University of New-Mexico, Albuquerque, NM, USA, leading research in opto- 650 electronics at the Center for High Technology Materials. Since October 2012, he 651 has been working with Telecom Paristech (alias École Nationale Supérieure des 652 Télécommunications), Paris, France, where he became an Associate Professor 653 then a Full Professor in January 2017. Since August 2015, he has also been serv- 654 ing as a Research Professor at the University of New-Mexico. He is the author 655 or coauthor of 77 journal papers, 1 book, 3 book chapters, and more than 170656 contributions in international conferences and workshops. His current research 657 interests include advanced quantum confined devices using new materials such 658 as quantum dots and dashes, light emitters based on intersubband transitions, 659 nonlinear dynamics and optical chaos in semiconductor lasers systems, as well 660 as microwave and silicon photonics applications including photonic clocks and 661 photonic analog-to-digital converters. He is an Associate Editor for Optics Ex- 662 press, a Senior Member of the SPIE and the IEEE Photonics Society, as well as 663 a Member of the OSA. 
666

NOTICES

667

668

669

670

671

672

673

674

675

676

677

678

679

680

681
- Author: If you have not completed your electronic copyright form (ECF) and payment option please return to Scholar One "Transfer Center." In the Transfer Center you will click on "Manuscripts with Decisions" link. You will see your article details and under the "Actions" column click "Transfer Copyright." From the ECF it will direct you to the payment portal to select your payment options and then return to ECF for copyright submission.

- Author: Please be aware that authors are required to pay overlength page charges $\$ 220$ per page if the paper is longer than 8 pages for Contributions and +12 pages for Invited Papers. If you cannot pay any or all of these charges please let us know.

- Author: This pdf contains 2 proofs. The first half is the version that will appear on Xplore. The second half is the version that will appear in print. If you have any figures to print in color, they will be in color in both proofs.

- Author: The option to publish your paper as Open Access expires when the paper is fully published on Xplore. Only papers published in "Early Access" may be changed to Open Access.

QUERIES

Q1. Author: If you have not completed your electronic copyright form (ECF) and payment option please return to Scholar One "Transfer Center". In the Transfer Center you will click on "Manuscripts with Decisions" link. You will see your article details and under the "Actions" column click "Transfer Copyright". From the ECF it will direct you to the payment portal to select your payment options and then return to ECF for copyright submission. 